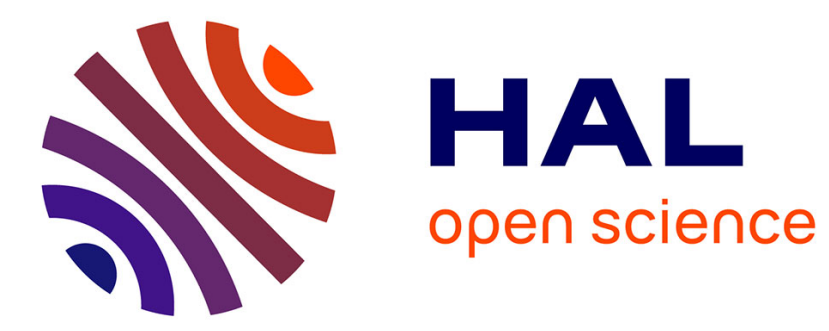

\title{
Historical mining and smelting in the Vosges Mountains (France) recorded in two ombrotrophic peat bogs
}

Benoît Forel, Fabrice Monna, Christophe Petit, Olivier Bruguier, R. Losno, P. Fluck, C. Begeot, H. Richard, V. Bichet, C. Chateau

\section{- To cite this version:}

Benoît Forel, Fabrice Monna, Christophe Petit, Olivier Bruguier, R. Losno, et al.. Historical mining and smelting in the Vosges Mountains (France) recorded in two ombrotrophic peat bogs. Journal of Geochemical Exploration, 2010, 107 (1), pp.9-20. 10.1016/j.gexplo.2010.05.004 . hal-00512409

\author{
HAL Id: hal-00512409 \\ https://hal.science/hal-00512409
}

Submitted on 15 Jun 2020

HAL is a multi-disciplinary open access archive for the deposit and dissemination of scientific research documents, whether they are published or not. The documents may come from teaching and research institutions in France or abroad, or from public or private research centers.
L'archive ouverte pluridisciplinaire HAL, est destinée au dépôt et à la diffusion de documents scientifiques de niveau recherche, publiés ou non, émanant des établissements d'enseignement et de recherche français ou étrangers, des laboratoires publics ou privés. 


\title{
Historical mining and smelting in the Vosges Mountains (France) recorded in two ombrotrophic peat bogs
}

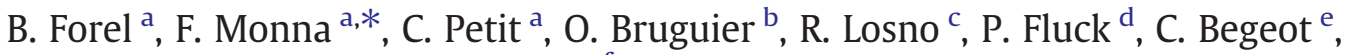 \\ H. Richard ${ }^{\mathrm{e}}$, V. Bichet ${ }^{\mathrm{e}}, \mathrm{C}$. Chateau ${ }^{\mathrm{f}}$ \\ a Laboratoire ARTéHIS, UMR 5594 CNRS, Culture Université de Bourgogne, UFR SVTE, 6 bd Gabriel, F-21000 Dijon, France \\ b Géosciences Montpellier, UMR 5243 CNRS, Université Montpellier 2, CC 60, Place Eugène Bataillon, F-34095 Montpellier Cedex 5, France \\ c LISA, Universités Paris 7, Paris 12, CNRS, Faculté des Sciences, 61 av. du Gal de Gaulle F-94010 Créteil Cedex, France \\ d CRESAT, UHA, 10 rue des frères Lumière, F-68093 Mulhouse Cedex, France \\ e Laboratoire Chrono-Environnement, UMR 6249 CNRS, Université de Franche-Comté, UFR Sciences et Techniques, 16 route de Gray F-25030 Besançon Cedex, France \\ ${ }^{\mathrm{f}}$ Université de Bourgogne, UFR SVTE, 6 bd Gabriel, F-21000 Dijon, France
}

\section{A R T I C L E I N F O}

\section{Article history:}

Received 19 November 2009

Accepted 20 May 2010

Available online 4 June 2010

\section{Keywords: \\ Environment \\ Pollution \\ Geochemistry \\ Archaeology \\ Lead isotopes \\ Peat \\ Mining \\ Metal \\ Vosges \\ Medieval}

\begin{abstract}
A B S T R A C T
Two peat sequences were sampled in the vicinity of the main mining districts of the Vosges Mountains: Sainte-Marie-aux-Mines and Plancher-les-Mines. Lead isotopic compositions and excess lead fluxes were calculated for each of these radiocarbon-dated sequences. Geochemical records are in very good agreement with the mining history of the area, well known over the last millennium. Except for an anomaly corresponding to the Middle Bronze Age which has not yet been resolved, there is no clear geochemical evidence of local metal production in the Vosges before the 10th century as excess lead deposition archived between $500 \mathrm{BC}$ and $500 \mathrm{AD}$ is attributed to long-range transport of polluted particulate matter. The approach described here can be applied to other mining districts where archaeological evidence is scarce or even lacking, but where past exploitation is suspected.(
\end{abstract}

\section{Introduction}

The crystalline Vosges Mountains (Northeast France) are well known for the abundant mineral resources (lead, silver, copper, and iron) they have provided through the ages. Metal production is attested from the 10th century in the district of Sainte-Marie-auxMines, which quickly became a rich and thriving city (Fluck, 2000). During the 15th and 16th centuries, the whole Vosges massif was intensively prospected and mined wherever possible. The locally abundant silver, which at that time was of more value than gold, was the main target. In $1545 \mathrm{AD}$, the Val d'Argent (or Silver Valley), located around Sainte-Marie-aux-Mines, counted 3000 miners, 600 mines and prospection areas, 12 smelters, and $150 \mathrm{~km}$ of galleries (Fluck and Weil, 1975). The history of metal production is nevertheless erratic, marked both by success stories and by declining profitability related to market competition, wars and natural catastrophes, wood supply and

\footnotetext{
* Corresponding author. Tel.: + 33380396 360; fax: + 33380396387. E-mail address: Fabrice.Monna@u-bourgogne.fr (F. Monna).
}

other technical constraints (Fluck, 2000). The operations finally stopped in the mid-20th century after a millennium of documented activity. Many galleries, tailings and wastes are still visible in the landscape. They clearly testify to the magnitude of this rich industrial past.

The most recent periods of activity are known from field evidence (more particularly at Sainte-Marie-aux-Mines where major archaeological work has been undertaken by Fluck and colleagues), written archives, and abundant iconographic documentation (e.g. the exceptional paintings by Heinrich Groff ca. 1530 AD, reproduced in Bari et al., 1992). However, the emergence and development of mining in the region are still not fully understood. Possible pioneer works starting from Antiquity or earlier are sometime evoked but no definitive answer can be given because field evidence is lacking. Such works may have been masked or irreparably destroyed. Recently, techniques originally developed by geoscientists have been adapted to reduce these gaps in archaeological knowledge (Mighall et al., 2002, 2009; Monna et al., 2004a,b; Arnaud et al., 2004; Baron et al., 2005). The basic idea is that if mining has taken place, it will have introduced significant quantities of metals into the surrounding area. Such environmental 
changes are therefore able to supply indirect evidence, provided that they have been well preserved over time. Ombrotrophic peatlands may fulfil such requirements. They are almost exclusively fed by the atmosphere so that mineral matter inputs are expected to be low (Shotyk, 1996a,b) and they are supposed to be hydrologically isolated from the substratum. Finally, anthropogenic contributions can be more easily extracted from the raw geochemical signal, and hence interpreted in historical terms (Shotyk et al., 1998, 2003; Mighall et al., 2002; Kylander et al., 2005; Jouffroy-Bapicot et al., 2007; see also Bindler, 2006 for a review). Among all metals studied, lead is often the most informative because its affinity with organic matter tends to prevent post-depositional migration (Dumontet et al., 1990; Vile et al., 1999; Shotyk et al., 1997, 2003; Weiss et al., 1999). Lead was not necessarily exploited by prehistoric societies, but because it is associated to many ore deposits including copper, it can be regarded as a proxy for various types of mining.

In the present study, we aim to establish a continuous history of metal-related activities in the Vosges massif, from the first signs of metallurgy to the most recent periods. The exceptional richness of archaeological and historical records in the region is a considerable

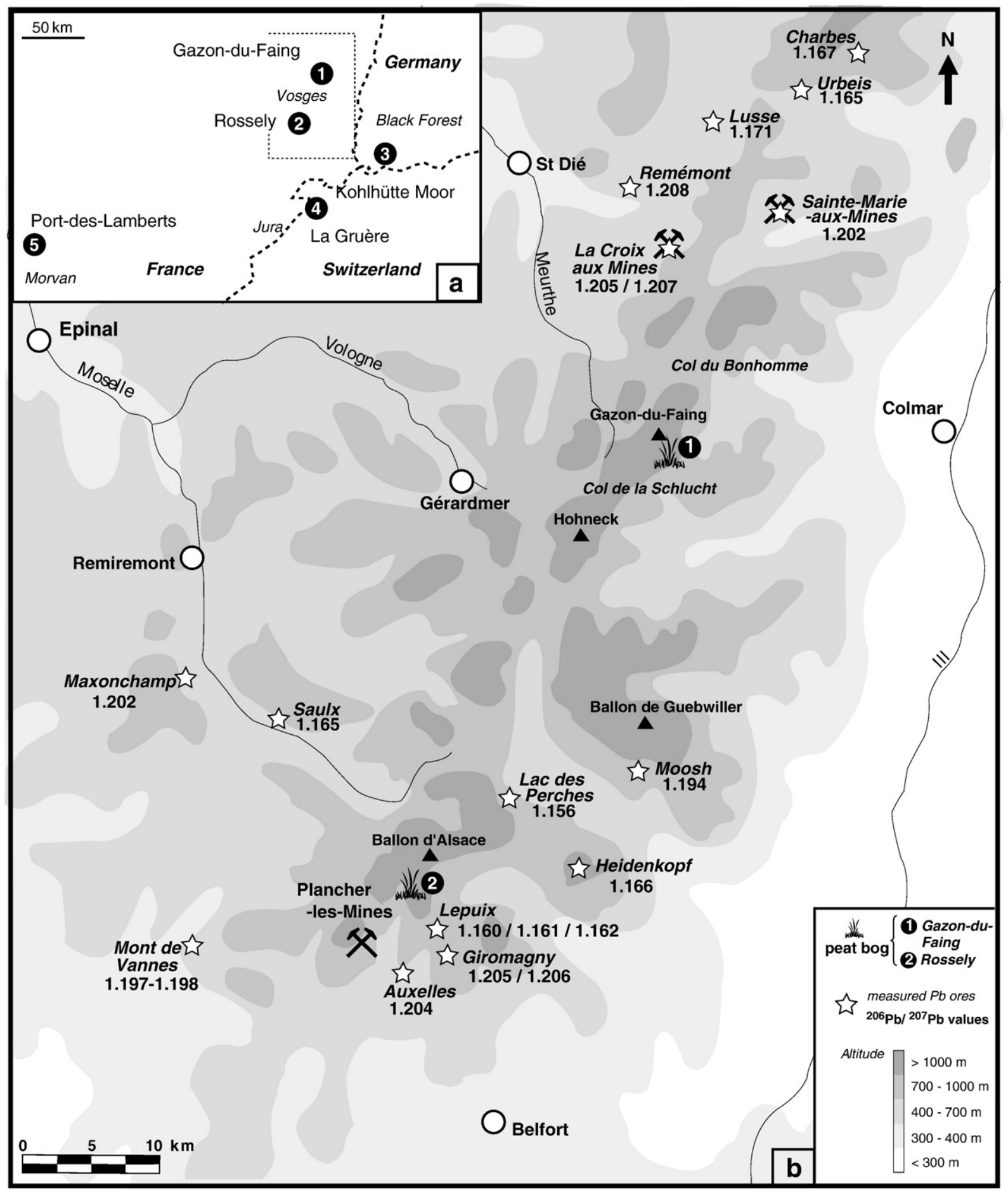

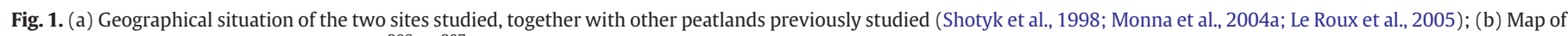
Vosges Mountains and sampling sites. The ${ }^{206} \mathrm{~Pb} /{ }^{207} \mathrm{~Pb}$ ratios of some $\mathrm{Pb}$ ore deposits measured by Marcoux (1987) are also reported. 
Table 1

Quality control for the analysis of the Gazon-du-Faing core performed with an ICP-MS HP-4500. Limits of detection (LOD) are calculated on the basis of 3 times the standard deviation of 4 analytical blanks. Measured and certified values of SRMs NIST 1547 and BHVO.

\begin{tabular}{|c|c|c|c|c|c|c|c|}
\hline & & $\mathrm{Pb}$ & $\mathrm{Zn}$ & $\mathrm{Cu}$ & La & $\mathrm{Sr}$ & $\mathrm{Bi}$ \\
\hline LOD (ng g $\left.{ }^{-1}\right)$ & & 0.1 & 2 & 0.1 & 0.001 & 0.06 & 0.002 \\
\hline \multicolumn{8}{|c|}{ NIST $1547\left(\mu \mathrm{g} \mathrm{g}^{-1}\right)$} \\
\hline \multirow[t]{2}{*}{ Measured } & $\mathrm{a}$ & 0.94 & 21.3 & 3.8 & 9.1 & 55.4 & 0.050 \\
\hline & $\mathrm{b}$ & 0.95 & 22.1 & 3.6 & 8.3 & 50.8 & 0.060 \\
\hline Certified & & 0.87 & 17.9 & 3.7 & $9^{*}$ & 53 & - \\
\hline \multicolumn{8}{|c|}{ BHVO $\left(\mu g^{-1}\right)$} \\
\hline Measured & & 1.95 & 104 & 130 & 15.3 & 393 & 0.019 \\
\hline Certified & & 2 & 105 & 136 & 15.2 & 400 & $0.018^{*}$ \\
\hline
\end{tabular}

*: provided but not certified.

Table 2

Quality control for the analysis of the Rossely core performed with a VG PQ2 +. Limits of detection (LOD) are calculated on the basis of 3 times the standard deviation of 4 blanks. Measured and certified values of NIST 1547.

\begin{tabular}{|c|c|c|c|c|c|c|}
\hline & & $\mathrm{Pb}$ & $\mathrm{Zn}$ & $\mathrm{Cu}$ & $\mathrm{La}$ & $\mathrm{Sr}$ \\
\hline $\operatorname{LOD}\left(\mathrm{ng} \mathrm{g}^{-1}\right)$ & & 0.1 & 1 & 0.2 & 0.005 & 0.2 \\
\hline \multicolumn{7}{|c|}{ NIST $1547\left(\mu \mathrm{g} \mathrm{g}^{-1}\right)$} \\
\hline \multirow[t]{3}{*}{ Measured } & $\mathrm{a}$ & 1.01 & 22.6 & 3.9 & 8.7 & 56.2 \\
\hline & b & 0.83 & 17.8 & 3.4 & 7.8 & 48.8 \\
\hline & c & 1.20 & 16.0 & 3.8 & 8.7 & 54.8 \\
\hline Certified & & 0.87 & 17.9 & 3.7 & 9* & 53 \\
\hline
\end{tabular}

*: provided but not certified.

asset which allows the potentialities of this palaeoenvironmental approach to be clearly evaluated over the last millennium. The two peat bogs studied, about $80 \mathrm{~km}$ apart, are located near the most important $\mathrm{Pb} / \mathrm{Zn} / \mathrm{Ag}$ mining districts in the Vosges: Sainte-Marie-auxMines and Plancher-les-Mines. Lead isotopes were measured in addition to elemental concentrations because they have proved to be a powerful tool to discriminate natural vs. anthropogenic lead in the environment, and even to distinguish between several anthropogenic sources (e.g. Faure, 1986; MacKenzie and Pulford, 2002; Mihaljevič et al., 2006; Kylander et al., 2009; Bindler et al., 2009; see also Komárek et al., 2008 for a complete review in the environment). It has been demonstrated that two populations of galena characterised by distinct lead isotopic compositions coexist in the Vosges Mountains (Marcoux, 1987). In such conditions, the use of lead isotopic compositions should be particularly informative.

\section{Materials and methods}

The peat deposits of Rossely $\left(06^{\circ} 48^{\prime} 01^{\prime \prime} \mathrm{E}-47^{\circ} 49^{\prime} 01^{\prime \prime} \mathrm{N}\right.$, altitude $920 \mathrm{~m}$ ) and Gazon-du-Faing $\left(07^{\circ} 03^{\prime} 10^{\prime \prime} \mathrm{E}-48^{\circ} 05^{\prime} 20^{\prime \prime} \mathrm{N}\right.$, altitude
$1220 \mathrm{~m}$ ) developed after glacial retreat (Fig. 1). They have a surface area of 10 ha and 9 ha, respectively. A Russian peat corer $(5 \mathrm{~cm}$ in diameter and $50 \mathrm{~cm}$ in length) was used to collect the sequences, following a twin-core coring (10 cm of overlap). The samples were wrapped in clean plastic bags immediately after collection in order to prevent external contamination. Master series were reconstructed at the laboratory on the basis of the sampling depths. Sub-sampling of the sequences, respectively 500 and $350 \mathrm{~cm}$ in length, was concentrated within the topmost $300 \mathrm{~cm}$ in the case of Rossely, and regularly spaced for Gazon-du-Faing. The sub-samples were dried for 2 days at $60{ }^{\circ} \mathrm{C}$ and powdered with a manual agate mortar to obtain homogeneous, fine powders. Water content (WC) was calculated taking into account humid and dried sample weights. Each sample was measured two or three times by elemental analyser at the CNRS Central Laboratory (Vernaison, France) for total organic carbon (TOC) and nitrogen contents. The results were averaged and uncertainties were typically of $\pm 5 \%$. Loss on ignition (LOI) was performed by ashing approximately $1 \mathrm{~g}$ of dried peat samples at $450{ }^{\circ} \mathrm{C}$ for $4 \mathrm{~h}$.

About $100 \mathrm{mg}$ of peat material was first oxidized with $2 \mathrm{~mL}$ of $\mathrm{HNO}_{3}$ of suprapure grade for 2-3 h on a hot plate. Complete digestion was then achieved under pressure on a hot plate using a mixture of $2 \mathrm{~mL}$ each of suprapure grade $\mathrm{HCl}, \mathrm{HNO}_{3}$ and $\mathrm{HF}$ (Merck, Germany). A one-third aliquot of solution was evaporated. The residues were retaken with $\mathrm{HNO}_{3}$ and precisely diluted with MilliQ water. Lanthanum, strontium, and lead contents were measured using two different quadrupole-based inductively coupled plasma-mass spectrometers (ICP-MS): a HP 4500 for the Gazon-du-Faing sequence (measurements also included bismuth contents), and a VG PQ2 + for the Rossely sequence. Blanks and certified reference materials (BHVO and Peach Leaves - NIST 1547) were prepared and added to the set of unknown samples following the same protocol (Tables 1-2). The concentrations measured for standard materials were within \pm 10 $15 \%$ of certified values. Uncertainties of $\pm 15 \%$ were therefore considered for all elements measured by ICP-MS. Lead from the remaining two-thirds aliquot was pre-concentrated on ionic resin AG1X4 (Biorad) and measured for isotopic abundance by Q-ICP-MS. Details regarding lead separation, instrumental settings, mass bias correction by bracketing, and dead time correction can be found elsewhere (Monna et al., 1998, 2000). The BCR-1, a certified reference material processed together with peat samples, yielded lead isotopic compositions $\left({ }^{206} \mathrm{~Pb} /{ }^{207} \mathrm{~Pb}=1.205 \pm 0.004\right.$ and ${ }^{208} \mathrm{~Pb} /{ }^{206} \mathrm{~Pb}=2.053 \pm$ 0.006 , at $95 \%$ confidence level) in good agreement with values reported in the literature $\left({ }^{206} \mathrm{~Pb} /{ }^{207} \mathrm{~Pb}=1.2036\right.$ and ${ }^{208} \mathrm{~Pb} /$ ${ }^{206} \mathrm{~Pb}=2.0582$; Baker et al., 2004). Blank corrections for isotopic measurements were never required because they appeared negligible compared to the total amount of lead in the aliquots. Six bulk samples for the Rossely core and five for the Gazon-du-Faing core were dated by radiocarbon (accelerator mass spectrometry) at the Poznań Radiocarbon Laboratory (Table 3). The dates were calibrated with the OxCal v.4.0 software (programme available for online use at https://c14.arch.ox.ac.uk/oxcal).

Table 3

Age-depth relationship for the cores of Rossely and Gazon-du-Faing. The data are calibrated to calendar dates AD or BC.

\begin{tabular}{|c|c|c|c|c|}
\hline Site & Bulk sample & Lab. reference & Uncalibrated ${ }^{14} \mathrm{C}$ date $( \pm 1 \sigma)$ & Calibrated calendar date $(2 \sigma)$ \\
\hline \multirow[t]{6}{*}{ Rossely } & ROS 61-63 & POZ-7322 & $740 \pm 35 \mathrm{BP}$ & [1220-1299]AD \\
\hline & ROS 103-105 & POZ-7193 & $1170 \pm 30 \mathrm{BP}$ & [778-902]AD \\
\hline & ROS 153-155 & POZ-7324 & $1605 \pm 35 \mathrm{BP}$ & [384-542]AD \\
\hline & ROS 203-205 & POZ-7194 & $2685 \pm 30 \mathrm{BP}$ & [897-802]BC \\
\hline & ROS 254-256 & POZ-7325 & $3240 \pm 40 \mathrm{BP}$ & [1617-1428]BC \\
\hline & ROS 305-307 & POZ-7195 & $3625 \pm 35 \mathrm{BP}$ & [2041-1884]BC \\
\hline \multirow[t]{5}{*}{ Gazon-du-Faing } & GDF 44-45 & POZ-17250 & $620 \pm 30 \mathrm{BP}$ & [1290-1400]AD \\
\hline & GDF 97-98 & POZ-17252 & $970 \pm 35 \mathrm{BP}$ & [990-1160]AD \\
\hline & GDF 159-160 & POZ-17253 & $1355 \pm 30 \mathrm{BP}$ & {$[620-770] A D$} \\
\hline & GDF 224-225 & POZ-17254 & $1845 \pm 35 \mathrm{BP}$ & [70-250]AD \\
\hline & GDF 285-286 & POZ-17255 & $2250 \pm 35 \mathrm{BP}$ & {$[400-200] B C$} \\
\hline
\end{tabular}




\section{Results and discussion}

\subsection{Growing rate of the peat sequences}

The age vs. depth relationship (Fig. 2) indicates fairly regular growing rates for each of the two sequences except, to some extent, for a relative slowing down between $\sim 150$ and $200 \mathrm{~cm}$ depth at the Rossely site. The sequences present no evident hiatus or major disturbance. The Rossely core covers more than four millennia, while the base of the Gazon-du-Faing core corresponds approximately to the transition between the Late Bronze Age and the Early Iron Age (ca. 8th century BC). Although the Gazon-du-Faing core is shorter than the Rossely core, the durations of both are adequate for our purpose.

\subsection{Trophic status of the sequences}

Both cores exhibit similar features with total organic carbon contents close to $50 \%$, ash content ca $2-3 \%$ (100-LOI\%), and
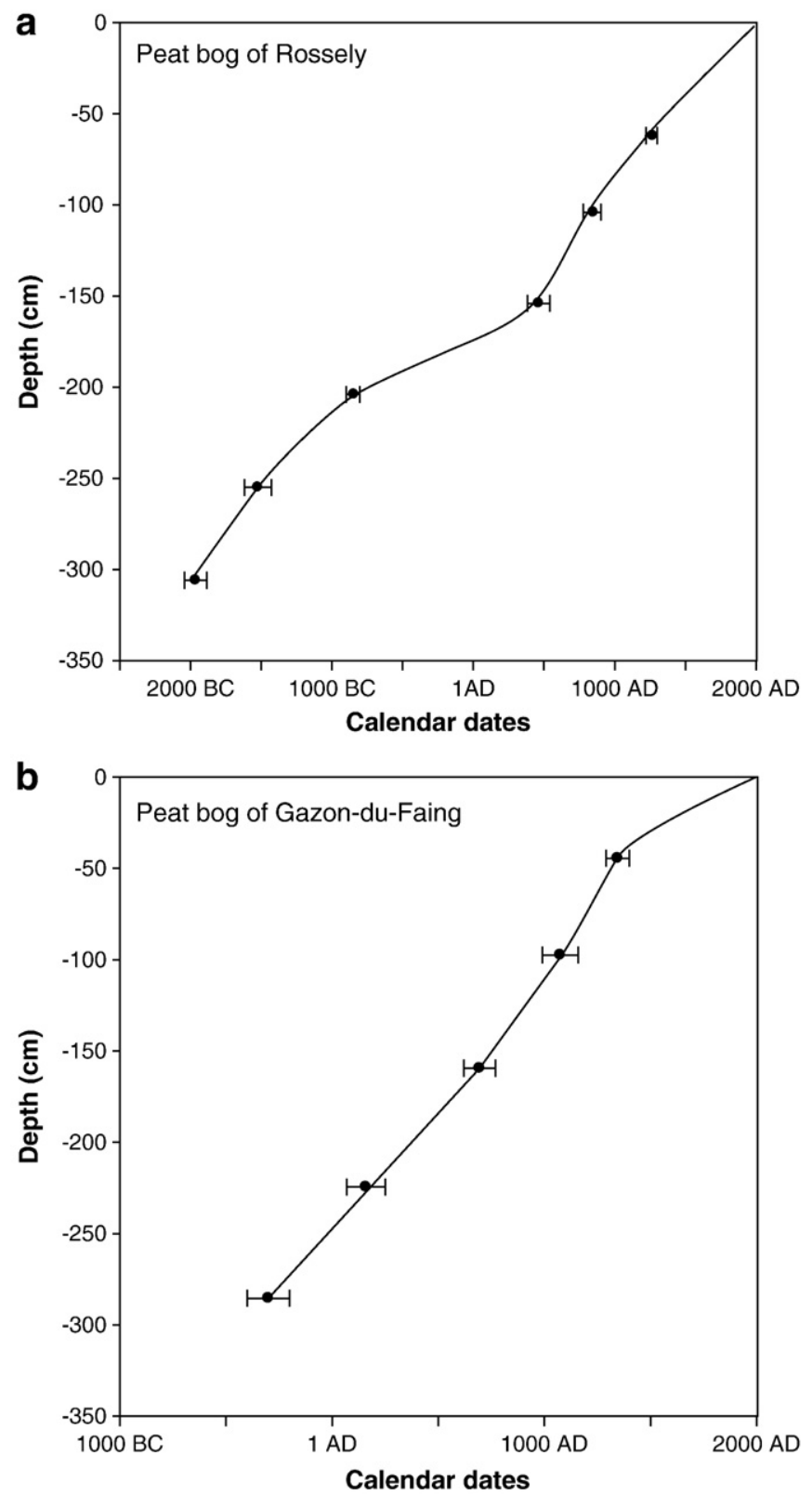

Fig. 2. Depth vs. ${ }^{14} \mathrm{C}$-based calibrated calendar dates for the peat bogs of Rossely (a) and Gazon-du-Faing (b). The error bars are given with a 95\% confidence level. concentrations of lanthanum (a lithogenic, conservative element belonging to the rare earth group, used as a surrogate for mineral matter) most of the time below $1 \mu \mathrm{g} \mathrm{g}^{-1}$. (Tables 4-5; Fig. 3a and b). The high $\mathrm{C} / \mathrm{N}$ ratios from 20 to more than 100 (Tables 4-5) confirm the visual examination of the sequences in the field: the sequences are homogeneous, and the organic matter is not strongly decomposed, even in deep horizons. The stable water content (w/w) with depth, at about $90 \%$ for Gazon-du-Faing and Rossely (not shown here), can easily be explained by the high water retention capacity of organic matter. All these features, as well as relatively steady $\mathrm{Sr}$ concentrations, below $20 \mathrm{\mu g} \mathrm{g}^{-1}$ (Tables 4-5; Fig. 3a and b), are characteristic of ombrotrophic status (e.g. Shotyk, 2002; De Vleeschouwer et al., 2007) well adapted to our purpose.

\subsection{Data preparation}

Even if both sequences are ombrotrophic, metal contributions of natural origin from the atmosphere cannot be excluded. While they are obviously of minor importance in the presence of large anthropogenic inputs, they constitute the heart of the signals observed during pre-anthropogenic times. Traditionally, the isolation of the excess metal contribution (assumed to be of anthropogenic nature) with respect to natural inputs is performed in two steps. In the first step, the targeted metal is normalised to a lithogenic and conservative element such as scandium, lanthanum, or aluminium, assuming that, in natural conditions, there is a proportional relationship between buried metals of natural origin (and present at trace levels) and the element used for normalisation (e.g. Martínez-Cortizas et al., 1997, 2002; Weiss et al., 2002; Monna et al., 2006). Then the excess contribution can be computed by using the following equation:

$M_{\text {excess }}=M_{\text {total }}-L a \times(M / L a)_{\text {natural }}$,

where $M_{\text {total }}$ represents the total metal $M$ concentration; $M_{\text {excess }}$, the excess contribution to the total concentration; La is the total lanthanum content, chosen here as a surrogate for natural mineral matter in this equation; $(\mathrm{M} / \mathrm{La})_{\text {natural }}$ is supposed to be constant and corresponds to the ratio between the metal $\mathrm{M}$ and lanthanum contents in pre-anthropogenic conditions.

Fig. $4 \mathrm{a}$ and $\mathrm{b}$ presents evolutions in total $\mathrm{Pb}$ concentrations and $\mathrm{Pb} / \mathrm{La}$ ratios and ${ }^{206} \mathrm{~Pb} /{ }^{207} \mathrm{~Pb}$ ratio profiles, for both cores. Because lanthanum contents do not vary widely with depth, the $\mathrm{Pb} / \mathrm{La}$ profiles are comparable to those of total $\mathrm{Pb}$. Below $300 \mathrm{~cm}$ depth in the Rossely core, $\mathrm{Pb} / \mathrm{La}$ ratios are low and relatively steady: $2.6 \pm 1.5(1 \sigma)$. Similar values are found at the base of the Gazon-du-Faing core: $2.0 \pm 0.7(1 \sigma)$, although it is not certain that the background was attained. These values are about 4-5 times greater than that the typical value of the upper continental crust $(\mathrm{Pb} / \mathrm{La} \sim 0.5$, Wedepohl, 1995), but should be preferred here as reference values, because they probably depict the local geochemical background better. Excess $\mathrm{Pb}$ profiles, computed using a $\mathrm{Pb} / \mathrm{La}$ ratio of $2.3 \pm 2(1 \sigma)$, for both cores, are shown in Fig. $4 \mathrm{a}$ and b. For each core, they appear to be almost the same as those of total lead because the natural contribution is too low to be significant. Surprisingly, below $300 \mathrm{~cm}$ depth in the Rossely core, in other words in horizons formed before $2000 \mathrm{BC}$, the ${ }^{206} \mathrm{~Pb} /{ }^{207} \mathrm{~Pb}$ ratios of $\sim 1.18$ (Table 4, Fig. 4a) are markedly lower than those generally observed for $\mathrm{Pb}$ naturally present at trace level in rocks and soils, supposed to be between 1.19 and 1.22 in France (Elbaz-Poulichet et al., 1986; Shotyk et al., 1998; Alfonso et al., 2001; Lévêque et al., 2002). It is nonetheless worth mentioning that values of 1.18-1.19 have already been reported by Le Roux et al. (2005) for lead isotopic compositions of preanthropogenic horizons in a peat column collected in the Black Forest, Germany, on the other side of the Rhine. Here, this signature probably reflects the local natural atmospheric deposition, as previously suggested for the Hercynian massif of the Black Forest by Le Roux et al. (2005). 
Table 4

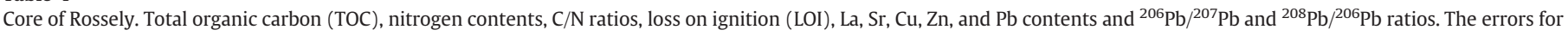
isotopic ratios are given at $95 \%$ confidence level. See text for errors in concentration measurements.

\begin{tabular}{|c|c|c|c|c|c|c|c|c|c|c|c|}
\hline Depth (cm) & TOC (\%) & $\mathrm{N}(\%)$ & $\mathrm{C} / \mathrm{N}$ & LOI (\%) & $\mathrm{La}\left(\mu \mathrm{g}^{-1}\right)$ & $\mathrm{Sr}\left(\mu \mathrm{g}^{-1}\right)$ & $\mathrm{Pb}\left(\mu \mathrm{g} \mathrm{g}^{-1}\right)$ & ${ }^{206} \mathrm{~Pb} /{ }^{207} \mathrm{~Pb}$ & \pm & ${ }^{208} \mathrm{~Pb} /{ }^{206} \mathrm{~Pb}$ & \pm \\
\hline-3 & 47.2 & 2.5 & 19 & - & 2.4 & 24.1 & 278 & 1.166 & 0.005 & 2.085 & 0.009 \\
\hline-6 & 47.4 & 2.2 & 22 & - & 1.0 & 13.6 & 133 & 1.179 & 0.009 & 2.093 & 0.013 \\
\hline-12 & 47.9 & 1.4 & 33 & 96.2 & 0.48 & 13.1 & 415 & 1.198 & 0.010 & 2.064 & 0.015 \\
\hline-18 & 48.1 & 1.2 & 42 & - & 0.32 & 11.9 & - & - & - & - & - \\
\hline-20 & - & - & - & - & 0.60 & 10.6 & 452 & 1.199 & 0.001 & 2.068 & 0.009 \\
\hline-24 & 47.5 & 0.77 & 62 & - & 1.5 & 4.0 & 283 & 1.195 & 0.005 & 2.065 & 0.014 \\
\hline-27 & 46.2 & 0.70 & 66 & - & 0.14 & 5.3 & 283 & 1.195 & 0.008 & 2.063 & 0.021 \\
\hline-30 & - & - & - & - & 0.51 & 9.4 & - & - & - & - & - \\
\hline-33 & 49.8 & 1.6 & 31 & - & 0.43 & 5.5 & 89 & - & - & - & - \\
\hline-39 & 49.3 & 1.0 & 48 & - & 0.80 & 8.1 & 48 & 1.197 & 0.008 & 2.078 & 0.016 \\
\hline-40 & - & - & - & - & 0.38 & 5.9 & 84 & 1.198 & 0.002 & 2.063 & 0.005 \\
\hline-45 & 49.8 & 1.3 & 37 & - & 0.61 & 10.0 & 17 & 1.185 & 0.007 & 2.078 & 0.020 \\
\hline-55 & 48.6 & 1.0 & 50 & - & 0.40 & 6.9 & 69 & 1.191 & 0.009 & 2.080 & 0.016 \\
\hline-59 & 49.1 & 0.88 & 56 & 98.0 & 0.20 & 6.5 & 106 & 1.193 & 0.006 & 2.064 & 0.016 \\
\hline-67 & 49.5 & 1.3 & 37 & - & 0.22 & 4.7 & 14 & 1.186 & 0.008 & 2.086 & 0.014 \\
\hline-71 & 48.5 & 0.88 & 55 & - & 0.24 & 8.1 & 15 & 1.175 & 0.006 & 2.090 & 0.017 \\
\hline-75 & 48.2 & 0.82 & 59 & - & 0.50 & 3.4 & 4.8 & - & - & - & - \\
\hline-78 & - & - & - & - & 0.21 & 4.1 & 4.6 & 1.180 & 0.004 & 2.085 & 0.005 \\
\hline-83 & 47.2 & 0.79 & 60 & 98.7 & 0.21 & 7.1 & 6.4 & - & - & - & - \\
\hline-87 & 48.7 & 0.89 & 55 & - & 0.23 & 8.3 & 7.7 & - & - & - & - \\
\hline-91 & 48.1 & 0.67 & 72 & - & 0.10 & 5.2 & 6.0 & - & - & - & - \\
\hline-95 & 49.8 & 0.95 & 52 & - & 0.20 & 3.8 & 5.5 & - & - & - & - \\
\hline-96 & - & - & - & - & 0.44 & 5.8 & 3.1 & 1.186 & 0.003 & 2.074 & 0.007 \\
\hline-107 & 48.5 & 0.88 & 55 & - & - & - & - & - & - & - & - \\
\hline-115 & - & - & - & - & 0.70 & 7.8 & 3.7 & 1.174 & 0.003 & 2.083 & 0.011 \\
\hline-119 & 48.9 & 0.99 & 49 & - & - & - & - & - & - & - & - \\
\hline-125 & 48.8 & 0.92 & 53 & 98.9 & 0.50 & 11.8 & 2.4 & 1.174 & 0.004 & 2.087 & 0.009 \\
\hline-137 & 48.6 & 1.2 & 42 & - & - & - & - & - & - & - & - \\
\hline-143 & 48.8 & 0.97 & 50 & - & - & - & - & - & - & - & - \\
\hline-146 & - & - & - & - & 0.54 & 6.1 & 2.8 & 1.166 & 0.003 & 2.094 & 0.006 \\
\hline-147 & 47.9 & 0.93 & 52 & - & - & - & - & - & - & - & - \\
\hline-153 & - & - & - & - & 0.57 & 5.6 & 2.4 & 1.174 & 0.003 & 2.087 & 0.007 \\
\hline-155 & 49.0 & 1.1 & 47 & 98.8 & - & - & - & - & - & - & - \\
\hline-161 & 51.1 & 1.5 & 35 & - & - & - & - & - & - & - & - \\
\hline-167 & 49.6 & 1.4 & 35 & - & - & - & - & - & - & - & - \\
\hline-173 & 50.9 & 1.9 & 27 & - & - & - & - & - & - & - & - \\
\hline-175 & - & - & - & - & 0.92 & 6.7 & 10 & 1.178 & 0.003 & 2.092 & 0.007 \\
\hline-179 & 51.4 & 1.8 & 28 & - & - & - & - & - & - & - & - \\
\hline-185 & - & - & - & - & 2.1 & 8.0 & 22 & 1.179 & 0.003 & 2.097 & 0.005 \\
\hline-191 & 51.6 & 0.71 & 73 & - & - & - & - & - & - & - & - \\
\hline-196 & - & - & - & - & 0.97 & 6.3 & 8.5 & 1.178 & 0.003 & 2.089 & 0.006 \\
\hline-197 & 50.7 & 1.1 & 46 & 98.6 & - & - & - & - & - & - & - \\
\hline-203 & - & - & - & - & 0.72 & 5.6 & 3.0 & 1.179 & 0.004 & 2.089 & 0.004 \\
\hline-207 & 48.5 & 0.81 & 60 & - & - & - & - & - & - & - & - \\
\hline-219 & 50.3 & 1.3 & 40 & - & - & - & - & - & - & - & - \\
\hline-225 & 50.1 & 0.90 & 56 & 98.6 & 0.68 & 6.7 & 0.9 & 1.183 & 0.005 & 2.084 & 0.009 \\
\hline-237 & 48.2 & 0.75 & 64 & - & - & - & - & - & - & - & - \\
\hline-243 & 49.4 & 0.81 & 61 & - & - & - & - & - & - & - & - \\
\hline-245 & - & - & - & - & 0.49 & 6.3 & 1.1 & 1.179 & 0.008 & 2.078 & 0.016 \\
\hline-247 & 48.0 & 0.66 & 73 & - & - & - & - & - & - & - & - \\
\hline-253 & - & - & - & - & 0.76 & 9.5 & 4.9 & 1.159 & 0.002 & 2.098 & 0.006 \\
\hline-258 & 48.4 & 0.73 & 66 & - & - & - & - & - & - & - & - \\
\hline-265 & - & - & - & - & 0.46 & 9.7 & 3.1 & 1.162 & 0.003 & 2.095 & 0.005 \\
\hline-270 & 48.5 & 0.74 & 66 & - & - & - & - & - & - & - & - \\
\hline-276 & 47.9 & 0.52 & 92 & - & - & - & - & - & - & - & - \\
\hline-282 & 47.4 & 0.46 & 103 & 98.9 & - & - & - & - & - & - & - \\
\hline-285 & - & - & - & - & 0.40 & 10.3 & 1.8 & 1.169 & 0.005 & 2.089 & 0.012 \\
\hline-294 & 46.6 & 0.63 & 74 & - & - & - & - & - & - & - & - \\
\hline-295 & - & - & - & - & 0.38 & 9.9 & 1.6 & 1.165 & 0.006 & 2.096 & 0.013 \\
\hline-302 & 48.9 & 0.32 & 153 & - & - & - & - & - & - & - & - \\
\hline-303 & - & - & - & - & 0.41 & 9.9 & 0.6 & 1.183 & 0.002 & 2.086 & 0.005 \\
\hline-315 & - & - & - & - & 0.39 & 12.1 & 0.9 & - & - & - & - \\
\hline-326 & 47.1 & 0.60 & 79 & 98.8 & & & & - & - & - & - \\
\hline-335 & - & - & - & - & 0.13 & 10.8 & 1.1 & 1.176 & 0.005 & 2.086 & 0.013 \\
\hline-345 & - & - & - & - & 0.20 & 11.8 & 1.0 & - & - & - & - \\
\hline-346 & 48.1 & 0.71 & 68 & 98.9 & - & - & - & - & - & - & - \\
\hline-360 & - & - & - & - & 0.20 & 14.0 & 0.4 & - & - & - & - \\
\hline-375 & 47.7 & 0.55 & 87 & - & - & - & - & - & - & - & - \\
\hline-389 & - & - & - & - & 0.15 & 15.8 & 0.7 & - & - & - & - \\
\hline-405 & 47.2 & 0.56 & 84 & 98.4 & - & - & - & - & - & - & - \\
\hline-430 & - & - & - & - & 0.18 & 20.4 & 0.4 & - & - & - & - \\
\hline-455 & 50.4 & 0.75 & 67 & - & - & - & - & - & - & - & - \\
\hline-485 & - & - & - & - & 0.71 & 6.9 & 0.6 & 1.186 & 0.009 & 2.076 & 0.018 \\
\hline-490 & 53.6 & 1.4 & 39 & 98.7 & - & - & - & - & - & - & - \\
\hline
\end{tabular}


Table 5

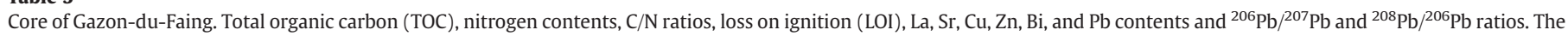
errors for isotopic ratios are given at $95 \%$ confidence level. See text for errors in concentration measurements.

\begin{tabular}{|c|c|c|c|c|c|c|c|c|c|c|c|c|}
\hline Depth $(\mathrm{cm})$ & TOC (\%) & $\mathrm{N}(\%)$ & $\mathrm{C} / \mathrm{N}$ & LOI (\%) & $\mathrm{La}\left(\mu \mathrm{g} \mathrm{g}^{-1}\right)$ & $\mathrm{Sr}\left(\mu \mathrm{g} \mathrm{g}^{-1}\right)$ & $\mathrm{Bi}\left(\mu \mathrm{g}^{-1}\right)$ & $\mathrm{Pb}\left(\mu \mathrm{g} \mathrm{g}^{-1}\right)$ & ${ }^{206} \mathrm{~Pb} /{ }^{207} \mathrm{~Pb}$ & \pm & ${ }^{208} \mathrm{~Pb} /{ }^{206} \mathrm{~Pb}$ & \pm \\
\hline-1 & - & - & - & - & 0.79 & 5.6 & 0.075 & 18 & 1.145 & 0.001 & 2.114 & 0.005 \\
\hline-6 & - & - & - & - & 1.5 & 4.6 & 0.089 & 37 & 1.130 & 0.001 & 2.128 & 0.004 \\
\hline-10 & 46.7 & 1.30 & 36 & 96.4 & - & - & - & - & - & - & - & - \\
\hline-15 & - & - & - & - & 2.1 & 15 & 0.541 & 158 & 1.165 & 0.002 & 2.096 & 0.005 \\
\hline-21 & 45.9 & 2.11 & 22 & - & - & - & - & - & - & - & - & - \\
\hline-26 & - & - & - & - & 1.8 & 7.3 & 0.094 & 46 & 1.184 & 0.002 & 2.080 & 0.006 \\
\hline-31 & 48.4 & 1.73 & 28 & 96.1 & - & - & - & - & - & - & - & - \\
\hline-36 & - & - & - & - & 0.79 & 6.9 & 0.027 & 17 & 1.193 & 0.002 & 2.070 & 0.005 \\
\hline-41 & - & - & - & - & 1.1 & 7.8 & 0.026 & 15 & 1.188 & 0.002 & 2.074 & 0.004 \\
\hline-47 & - & - & - & - & 1.3 & 11 & 0.037 & 16 & 1.181 & 0.002 & 2.078 & 0.006 \\
\hline-53 & 45.4 & 0.47 & 97 & - & - & - & - & - & - & - & - & - \\
\hline-58 & - & - & - & - & 0.53 & 10 & 0.101 & 18 & 1.181 & 0.002 & 2.083 & 0.006 \\
\hline-63 & - & - & - & - & 0.81 & 10 & 0.071 & 16 & 1.177 & 0.001 & 2.087 & 0.004 \\
\hline-68 & 46.1 & 0.33 & 140 & 98.3 & 0.65 & 9.8 & 0.045 & 17 & 1.175 & 0.001 & 2.090 & 0.006 \\
\hline-74 & - & - & - & - & 0.32 & 7.8 & 0.020 & 10 & - & - & - & - \\
\hline-81 & - & - & - & - & 0.66 & 11 & 0.021 & 17 & 1.186 & 0.002 & 2.076 & 0.005 \\
\hline-91 & 46.3 & 0.43 & 108 & - & 0.31 & 7.0 & 0.016 & 14 & 1.191 & 0.001 & 2.071 & 0.005 \\
\hline-96 & - & - & - & - & 0.92 & 9.3 & 0.018 & 14 & 1.190 & 0.002 & 2.070 & 0.004 \\
\hline-103 & - & - & - & - & 0.44 & 8.8 & 0.016 & 7.8 & 1.180 & 0.002 & 2.082 & 0.005 \\
\hline-108 & 44.8 & 0.32 & 140 & 98.7 & - & - & - & - & - & - & - & - \\
\hline-113 & - & - & - & - & 0.77 & 9.9 & 0.013 & 2.5 & 1.180 & 0.001 & 2.086 & 0.004 \\
\hline-118 & - & - & - & - & 0.36 & 6.1 & 0.007 & 1.6 & 1.176 & 0.002 & 2.091 & 0.005 \\
\hline-123 & - & - & - & - & 0.22 & 6.5 & 0.015 & 1.5 & 1.173 & 0.001 & 2.092 & 0.006 \\
\hline-129 & 46.3 & 0.20 & 232 & - & - & - & - & - & - & - & - & - \\
\hline-134 & - & - & - & - & 0.30 & 8.3 & 0.011 & 0.91 & 1.173 & 0.002 & 2.097 & 0.004 \\
\hline-139 & - & - & - & - & 0.26 & 8.4 & 0.010 & 1.4 & 1.169 & 0.003 & 2.102 & 0.007 \\
\hline-144 & - & - & - & - & 0.35 & 13 & 0.009 & 1.0 & 1.174 & 0.002 & 2.094 & 0.003 \\
\hline-153 & 46.8 & 0.65 & 72 & 98.6 & 0.61 & 8.0 & 0.018 & 2.4 & 1.177 & 0.002 & 2.086 & 0.004 \\
\hline-158 & - & - & - & - & 0.26 & 7.2 & 0.010 & 1.6 & 1.169 & 0.001 & 2.101 & 0.004 \\
\hline-168 & 44.7 & 0.10 & 447 & - & 0.20 & 7.6 & 0.007 & 0.6 & 1.168 & 0.003 & 2.108 & 0.004 \\
\hline-173 & - & - & - & - & 0.39 & 7.9 & 0.006 & 1.4 & 1.167 & 0.001 & 2.109 & 0.005 \\
\hline-178 & 47.0 & 0.27 & 174 & 99.1 & 0.38 & 8.8 & 0.008 & 0.94 & - & - & - & - \\
\hline-183 & - & - & - & - & 0.28 & 7.8 & 0.018 & 0.82 & 1.168 & 0.003 & 2.111 & 0.007 \\
\hline-188 & - & - & - & - & 0.47 & 8.5 & 0.016 & 1.6 & 1.172 & 0.002 & 2.096 & 0.002 \\
\hline-193 & - & - & - & - & 0.46 & 8.3 & 0.012 & 1.2 & 1.171 & 0.002 & 2.101 & 0.006 \\
\hline-198 & 47.5 & 0.72 & 66 & - & 0.42 & 6.7 & 0.012 & 1.4 & 1.172 & 0.002 & 2.098 & 0.005 \\
\hline-208 & 47.9 & 0.45 & 106 & 98.9 & 0.44 & 7.0 & 0.011 & 4.9 & - & - & - & - \\
\hline-213 & - & - & - & - & 0.24 & 5.2 & 0.011 & 7.3 & 1.157 & 0.001 & 2.098 & 0.005 \\
\hline-218 & - & - & - & - & 0.39 & 5.9 & 0.010 & 4.0 & 1.165 & 0.002 & 2.099 & 0.005 \\
\hline-223 & 46.3 & 0.19 & 244 & 99.0 & - & - & - & - & - & - & - & - \\
\hline-228 & - & - & - & - & 0.56 & 9.0 & 0.007 & 6.4 & 1.174 & 0.002 & 2.068 & 0.004 \\
\hline-238 & - & - & - & - & 0.53 & 8.7 & 0.016 & 8.0 & 1.171 & 0.002 & 2.095 & 0.005 \\
\hline-243 & - & - & - & - & 0.58 & 8.9 & 0.041 & 11 & 1.169 & 0.002 & 2.096 & 0.003 \\
\hline-248 & 45.8 & 0.30 & 153 & 98.8 & 0.47 & 9.5 & 0.021 & 8.7 & 1.174 & 0.002 & 2.094 & 0.004 \\
\hline-259 & - & - & - & - & 0.46 & 8.8 & 0.034 & 13 & 1.171 & 0.001 & 2.093 & 0.005 \\
\hline-264 & 46.6 & 0.91 & 51 & - & - & - & - & - & - & - & - & - \\
\hline-269 & - & - & - & - & 0.42 & 10 & 0.018 & 12 & 1.171 & 0.001 & 2.093 & 0.004 \\
\hline-274 & - & - & - & - & 0.36 & 10 & 0.012 & 9.2 & 1.171 & 0.001 & 2.097 & 0.005 \\
\hline-279 & - & - & - & - & 0.44 & 11 & 0.013 & 11 & 1.174 & 0.002 & 2.092 & 0.004 \\
\hline-284 & 48.1 & 0.25 & 193 & 98.5 & 0.74 & 10 & 0.025 & 11 & 1.174 & 0.002 & 2.097 & 0.004 \\
\hline-289 & - & - & - & - & 0.58 & 11 & 0.018 & 7.0 & 1.174 & 0.001 & 2.092 & 0.004 \\
\hline-294 & 48.1 & 0.72 & 67 & - & 0.73 & 11 & 0.025 & 4.6 & 1.178 & 0.001 & 2.093 & 0.005 \\
\hline-299 & - & - & - & - & 0.83 & 11 & 0.024 & 4.3 & 1.173 & 0.001 & 2.092 & 0.006 \\
\hline-308 & - & - & - & - & 0.57 & 12 & 0.012 & 1.4 & 1.174 & 0.002 & 2.096 & 0.006 \\
\hline-314 & 45.7 & 0.39 & 117 & 98.7 & 0.37 & 14 & 0.009 & 2.5 & 1.160 & 0.001 & 2.100 & 0.006 \\
\hline-320 & - & - & - & - & 0.28 & 8.6 & 0.008 & 0.76 & 1.174 & 0.002 & 2.097 & 0.004 \\
\hline-333 & - & - & - & - & 0.78 & 15 & 0.015 & 1.3 & 1.171 & 0.002 & 2.096 & 0.005 \\
\hline-339 & 50.5 & 1.53 & 33 & 98.0 & 0.94 & 12 & 0.020 & 1.4 & 1.177 & 0.002 & 2.097 & 0.006 \\
\hline
\end{tabular}

Comparisons of excess lead profiles between cores are nonetheless difficult even when a chronological scale is preferred to a depth scale because concentrations are directly governed by growth rates of peatlands. For this reason, fluxes of excess lead were calculated for the Rossely and Gazon-du-Faing sequences by taking into account excess lead contents, accumulation rates and apparent densities (cf Mihaljevič et al. 2006). The two sequences for the Vosges are reported together with three others collected in surrounding areas (Fig. 1a, Fig. 5): (i) the "Kohlhütte Moor" sequence, located south of the Black Forest, Germany, about $90 \mathrm{~km}$ from our sites (Le Roux et al., 2005), (ii) the "Etang de la Gruère" sequence, in the Swiss Jura mountains, $80 \mathrm{~km}$ away (data in Shotyk et al., 1998, fluxes reported in Le Roux et al., 2005), and (iii) the "Port-des-Lamberts" sequence, situated in the Morvan, Massif Central, France, $240 \mathrm{~km}$ away (data in Monna et al., 2004a). The ${ }^{206} \mathrm{~Pb} /{ }^{207} \mathrm{~Pb}$ ratios of the average anthropogenic component alone were calculated when possible using the procedure described in Monna et al. (2004a), which takes into account the ${ }^{206} \mathrm{~Pb} /{ }^{207} \mathrm{~Pb}$ and $\mathrm{La} / \mathrm{Pb}$ ratios (or $\mathrm{Sc} / \mathrm{Pb}$ ratios for the Gruère core) of both background and bulk samples.

\subsection{Reading lead fluxes and lead isotopic compositions in historical terms}

\subsubsection{Prehistoric times-Bronze Age}

Before $2000 \mathrm{BC}$, no measurable anthropogenic inputs can be recognized in the Rossely sequence (Fig. 5b). From 2000 BC to 

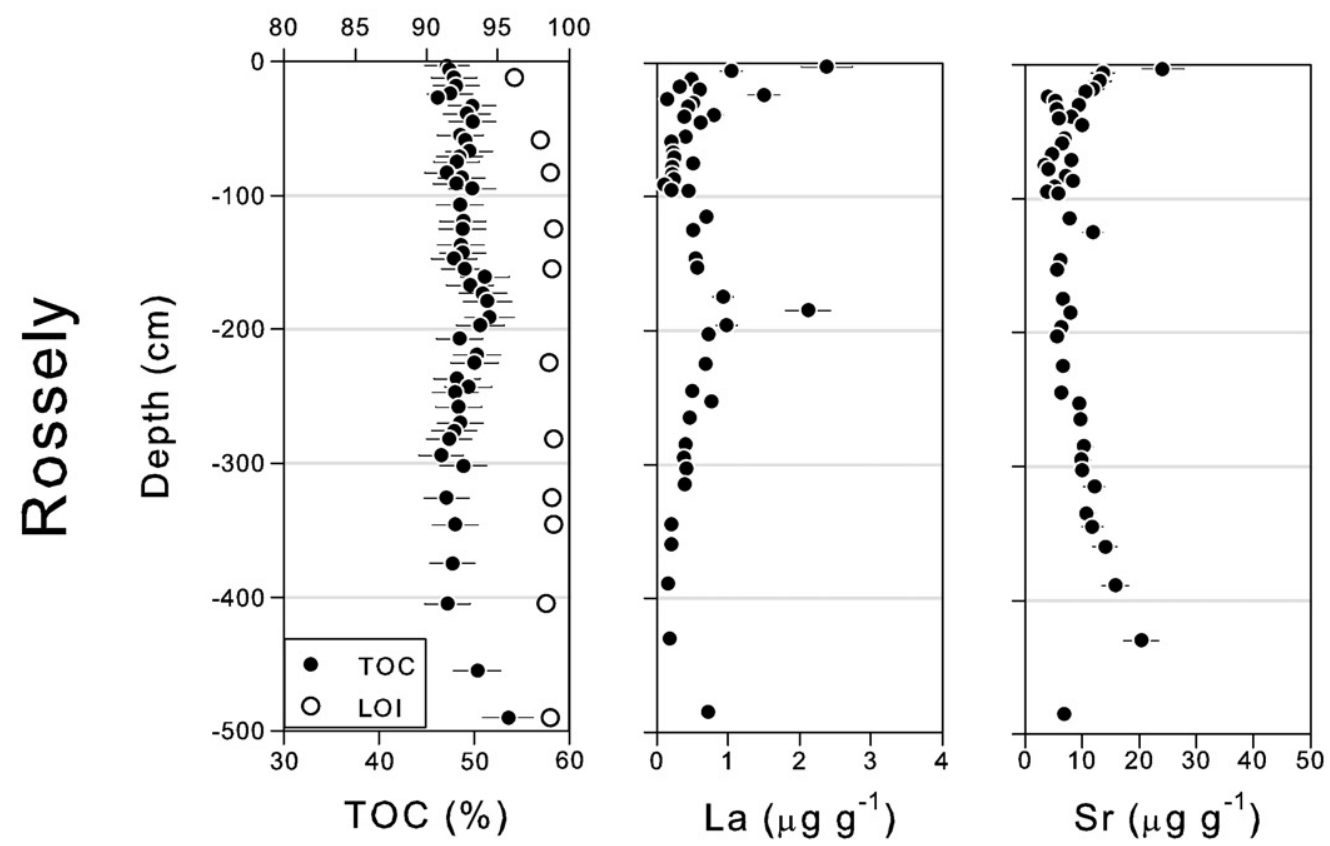

\section{LOI $(\%)$}
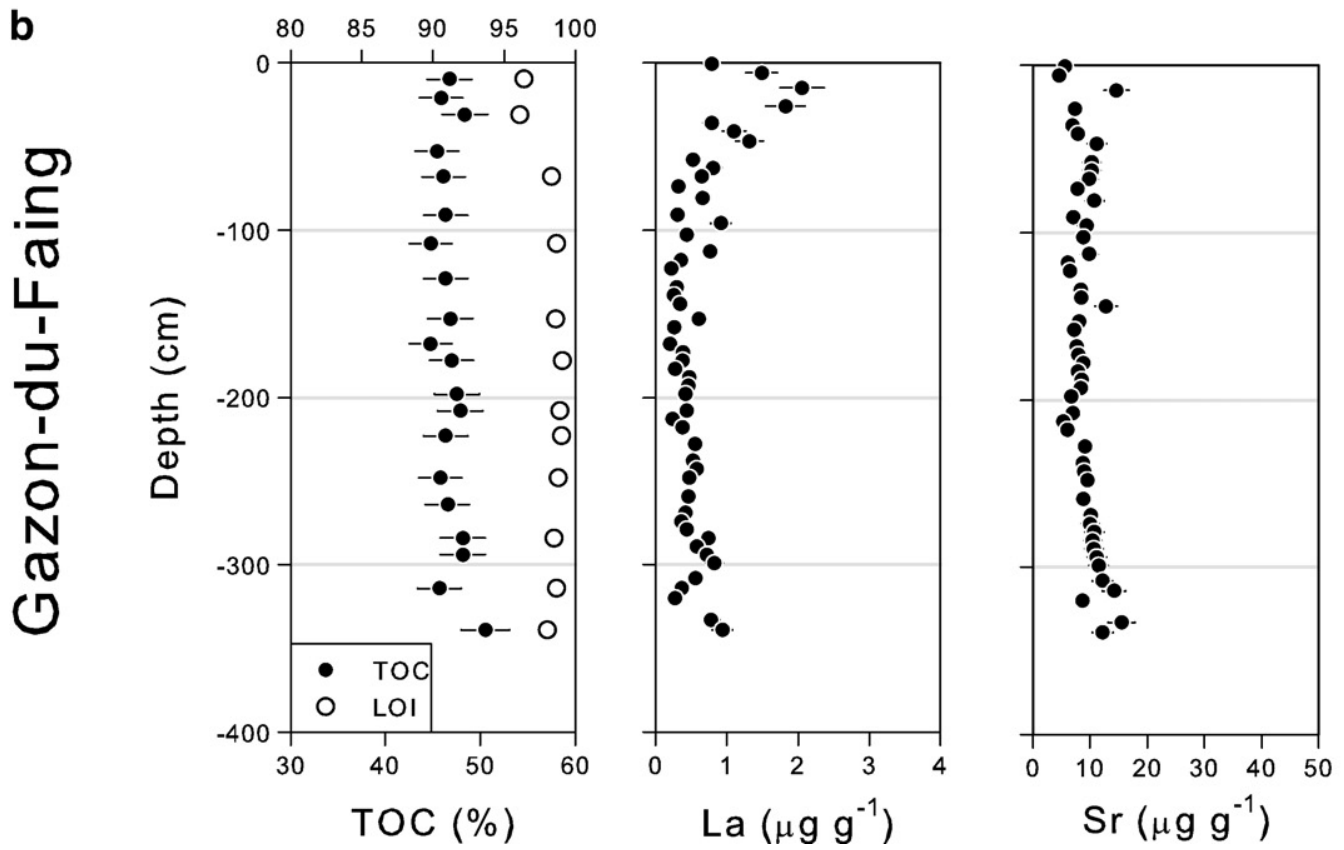

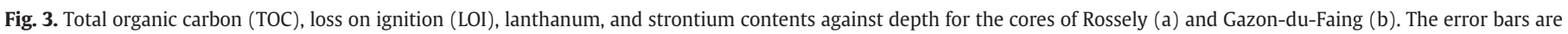
given with a $95 \%$ confidence level.

$1500 \mathrm{BC}$, discrete but significant geochemical anomalies with excess $\mathrm{Pb}$ fluxes reaching $0.2 \mathrm{mg} \mathrm{m}^{-2} \mathrm{y}^{-1}$ are noticed in the Rossely sequence, although nothing similar has been seen in the Morvan core, which covers the same period (Fig. 5e). This variation could perhaps be attributed to a durable episode of changes in the nature of the mineral matter integrating the peatland, in other words changes in $\mathrm{Pb} / \mathrm{La}$ ratios. This hypothesis is however not fully satisfactory because the lead isotopic signature of excess lead $\left({ }^{206} \mathrm{~Pb} /{ }^{207} \mathrm{~Pb} \sim 1.155\right)$ is incompatible with natural dust inputs. Another possible explanation could be local mining by prehistoric societies, for which no archaeological evidence remains. This scenario is compatible with the lead isotopic compositions of one group of galena extracted from the surrounding ore deposits, which present ${ }^{206} \mathrm{~Pb} /{ }^{207} \mathrm{~Pb}$ ratios 1.15-1.16 (see Fig. 1 for localisation and Fig. 6, details are given in Table 6). However, the available data are too scarce to confirm either hypothesis. 

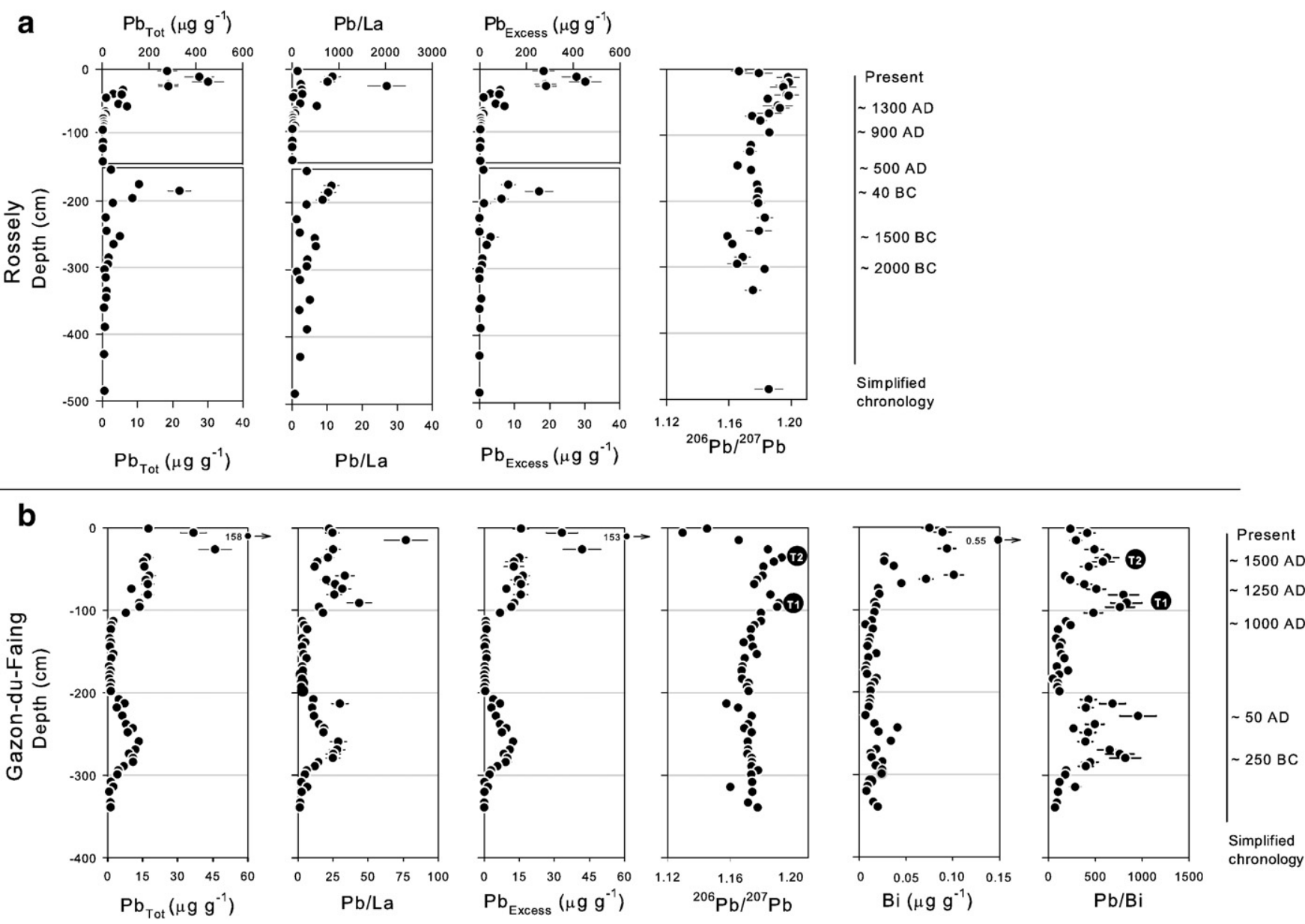

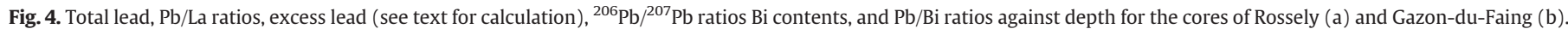
The error bars are given with a $95 \%$ confidence level.

\subsubsection{Bronze Age-Antiquity}

In the Rossely core, the period between $1300 \mathrm{BC}$ and $600 \mathrm{BC}$ is characterised by extremely low excess Pb fluxes (Fig. 5b). In contrast, the Morvan core recorded notable anthropogenic inputs during this period (Fig. 5e) which were interpreted as the impact of mining and metallurgy performed by nearby populations (Monna et al., 2004a). Significant excess $\mathrm{Pb}$ fluxes are noticeable in the Vosges during the Iron Age and Antiquity (Fig. 5a and b). It is well established that, during this period, mining and metallurgy developed in Europe (Settle and Patterson, 1980; Nriagu, 1983, 1996). The amount of lead introduced into the atmosphere was so large that significant fall-out of anthropogenic origin is systematically recognized in lake sediments and peat bogs across Europe (e.g. Shotyk et al., 1998; Kylander et al., 2009) reaching areas as remote as Sweden or Greenland where metallurgy was obviously not present at that time (Hong et al., 1994; Rosman et al., 1997; Brännvall et al., 1999). Because the contamination was widespread, some authors have even proposed using the lead anomaly as a chronostratigraphic marker to date sediments (Renberg et al., 2001). It is therefore not surprising to observe this signal in the Vosges Mountains. The point is nonetheless to determine if lead emitted by possible local mining may have been added in substantial amounts to that resulting from long-range transport of polluted airborne particulate matter. Comparing lead isotopic signatures of anthropogenic lead contribution and fluxes recorded in the Vosges Mountains with other neighbouring sites can help to answer this question. First of all, the anthropogenic lead flux peaked at approximately the same time in the Vosges, Morvan, Black Forest and Swiss Jura. Time shifts can be observed between the profiles but they may be due to uncertainties in the chronology, more particularly at Rossely where the time scale is poorly constrained between $1000 \mathrm{BC}$ and $1000 \mathrm{AD}$. In addition, moderate postdeposition migrations of $\mathrm{Pb}$ may have led to an overestimation of the contamination phase duration. There is a remarkable similarity in the maximum anthropogenic lead fluxes: about $1 \mathrm{mg} \mathrm{m}^{-2} \mathrm{y}^{-1}$ at each of these sites, although metallic deposition is known to be controlled by local climatic and geomorphological conditions (rainfall amount, topography, etc.). However, while the isotopic signatures of the anthropogenic component were approximately the same at Rossely $\left({ }^{206} \mathrm{~Pb} /{ }^{207} \mathrm{~Pb} \sim 1.178-1.179\right.$, Fig. 5b), Gazon-du-Faing $\left({ }^{206} \mathrm{~Pb} /\right.$ ${ }^{207} \mathrm{~Pb} \sim 1.174-1.175$; Fig. 5a) and in the Jura $\left({ }^{206} \mathrm{~Pb} /{ }^{207} \mathrm{~Pb} \sim 1.176-\right.$ 1.177; Fig. 5d), a slightly less radiogenic component was noticed in the Morvan during this period $\left({ }^{206} \mathrm{~Pb} /{ }^{207} \mathrm{~Pb} \sim 1.170-1.172\right.$, Fig. $\left.5 \mathrm{e}\right)$; no isotopic data is available for the Black Forest core during Antiquity (Fig. 5c). These features plead in favour of a common remote source impacting all sites in approximately the same way. The only difference resides in the situation of the Morvan where the adjunction of locally derived pollution may have produced a mean anthropogenic component slightly less radiogenic than those calculated for the other sites. Everything therefore tends to demonstrate that, despite the abundance of mineral resources, significant local mining did not take place in the Vosges at this period.

\subsubsection{Middle Ages-Modern times}

Excess lead appears to be low in all cores including those from the Vosges from the 4th century AD and for six centuries onwards (Fig. 5). 

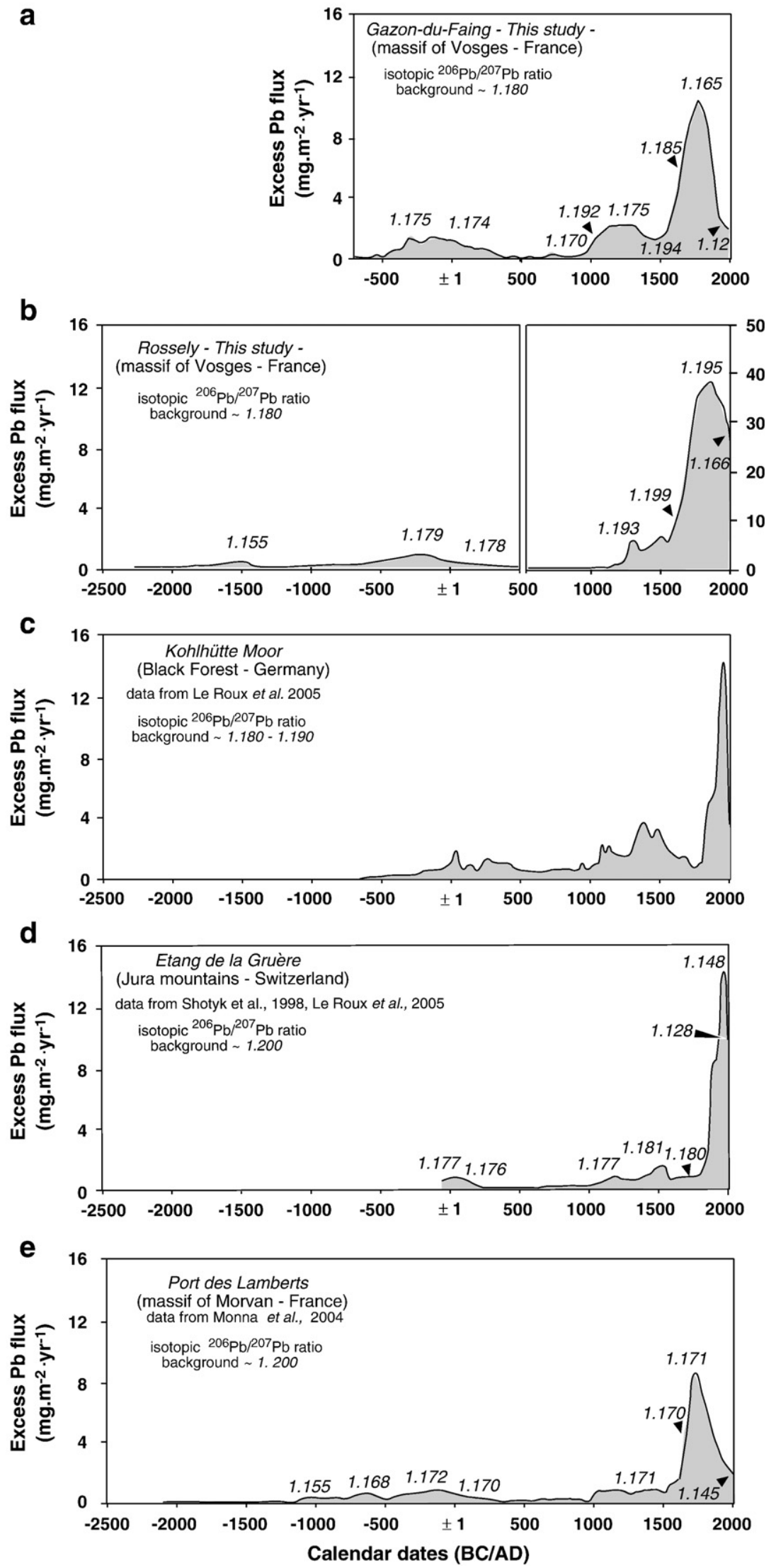

Fig. 5. Excess lead fluxes through time: Gazon-du-Faing (a) and Rossely (b) in the Vosges Moutains; Kohlhütte Moor (c) in the Black Forest; Etang de la Gruère (d) in the Swiss Jura Mountains; Port des Lamberts (e) in the Morvan massif. The isotopic ${ }^{206} \mathrm{~Pb} /{ }^{207} \mathrm{~Pb}$ ratios of excess lead are reported on the graphs. Attention must be paid to the Rossely diagram which presents a double $Y$-axis scale. 


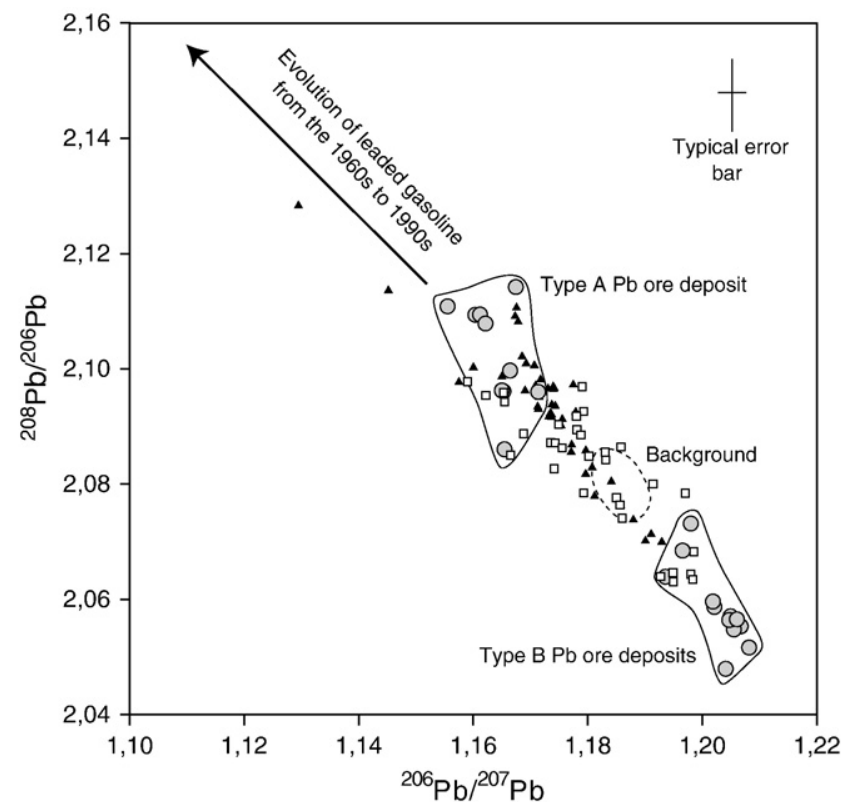

Fig. 6. ${ }^{206} \mathrm{~Pb} /{ }^{207} \mathrm{~Pb}$ vs. ${ }^{208} \mathrm{~Pb} /{ }^{206} \mathrm{~Pb}$ diagram. Open boxes and closed triangles correspond to samples from Rossely and Gazon-du-Faing respectively. Potential lead sources are reported for comparative purposes: Grey circles for local Pb ore deposits of types A and B (data from Marcoux, 1987), and recent evolution of leaded gasoline (see text for details). Typical error bars for isotopic measurements are also reported (95\% confidence level).

With the fall of the Roman Empire, a period known as the Dark Ages began. Many mines closed across Europe and the main metallurgical centres slowed down. Wars and barbarian invasions disorganized industrial production and airborne particulate matter became rapidly less contaminated at the European scale (Shotyk et al., 1998; Brännvall et al., 1999). From the 10th century to the present, both environmental records and the local history of mining and smelting differ somewhat for the districts of Sainte-Marie-aux-Mines and Plancher-les-Mines. As a consequence, they are discussed separately.

3.4.3.1. Gazon-du-Faing. A drastic increase in excess Pb deposition is noticed from the 10th century (Fig. 5a). This intensification is accompanied by a major shift in lead isotopic compositions towards more radiogenic values; the calculated ${ }^{206} \mathrm{~Pb} /{ }^{207} \mathrm{~Pb}$ ratios of excess lead progress rapidly from 1.170 to 1.192 . The oldest field evidence of mining ever found at Sainte-Marie-aux-Mines dates from that period. It is based on two radiocarbon analyses of coals (845-980 AD and 895-1015 AD) associated with scoria and furnace fragments (Fluck, 2000). Such dates are in very good agreement with the earliest written source which reports that the monk who founded the monastery of Echery (ca. $938 \mathrm{AD}$ ) also started mining activities in the area (Fluck, 2000 and sources cited therein). Moreover, the rise of the ${ }^{206} \mathrm{~Pb} /{ }^{207} \mathrm{~Pb}$ ratios toward radiogenic values is coherent with the signatures of one group of local galena, namely type $\mathrm{B}$, which present ${ }^{206} \mathrm{~Pb} /{ }^{207} \mathrm{~Pb}$ ratios of about 1.20 (see Fig. 1 for localisation and Fig. 6, details are given in Table 6). Lead isotopic compositions of peat undoubtedly record the environmental impact of local mining and smelting. From the 11th to the 13th centuries $A D$, excess lead fluxes stabilised, reaching more than $2 \mathrm{mg} \mathrm{m}^{-2} \mathrm{y}^{-1}$, while ${ }^{206} \mathrm{~Pb} /{ }^{207} \mathrm{~Pb}$ ratios of the anthropogenic lead signature regularly decreased to 1.175 . Smelters, galleries and hydrological installations built to wash ores, which all testify to persistent metallurgical activity from the 10th to 14th century AD have been discovered by Fluck and colleagues in the Altenberg (Sainte-Marie-auxMines district). The fall of the ${ }^{206} \mathrm{~Pb} /{ }^{207} \mathrm{~Pb}$ ratios observed in the Gazondu-Faing peat bog probably illustrates the progressive extension of mining works to another group of local ore deposits (i.e. galena of type A, characterised by ${ }^{206} \mathrm{~Pb} /{ }^{207} \mathrm{~Pb}$ ratios of about 1.165 , cf Table 6, Fig. 6). There is a turning point around the 13th-14th century. Lead pollution decreased and ${ }^{206} \mathrm{~Pb} /{ }^{207} \mathrm{~Pb}$ ratios of the pollutant end-member rose again to values greater than 1.190 (Fig. 5a). It is known that mining declined at that time without totally collapsing (Fluck, 2009) in part because of the disorganisation of the society due to successive famines (1292-1295 and 1315-1317), the Black Plague which was particularly severe in 1348, 1359, 1370 and 1400 in the Vosges, and the Hundred Years' War lasting from 1337 to 1453 . Other reasons, such as technical problems related to the accumulation of water in galleries, have also been invoked to explain this recession. Production is known to have focused only on specific mines selected for their profitability. Such a change is illustrated by the sudden shift in ${ }^{206} \mathrm{~Pb} /{ }^{207} \mathrm{~Pb}$ ratios toward more radiogenic values. From the beginning of the $16^{\text {th }}$ century, anthropogenic lead fluxes quickly increased to reach more than $10 \mathrm{mg} \mathrm{m}^{-2} \mathrm{y}^{-1}$ during the 17 th-18th century, while ${ }^{206} \mathrm{~Pb} /{ }^{207} \mathrm{~Pb}$ ratios of the anthropogenic contribution dropped to 1.165 (Fig. 5a). This new turning point is in perfect agreement with the revival of mining exploitation which occurred from the end of the 15th century AD at Sainte-Marie-aux-Mines. The first success recorded from the beginning of the 16th century AD led to intense and systematic prospection. However, difficulties quickly appeared: (i) forest clearances had to extend to respond to the increasing demand for shoring beams and the wood supply decreased so that its price rose, (ii) too much silver persisted in copper ingots reducing profits, and (iii) mined veins deepened and consequently the exploitation cost increased.

\section{Table 6}

Lead isotopic compositions of Vosges ore deposits measured by TIMS (data from Marcoux, 1987). Errors were typically $\pm 0.01, \pm 0.01$ and \pm 0.02 for ${ }^{206} \mathrm{~Pb} /{ }^{204} \mathrm{~Pb},{ }^{207} \mathrm{~Pb}$ ${ }^{204} \mathrm{~Pb},{ }^{208} \mathrm{~Pb} /{ }^{204} \mathrm{~Pb}$ ratios, respectively (at 95\% confidence level). Ak, G, Bl, Z, P, Bert., for aikinite, galena, boulangerite, zinkenite, plagionite, and berthierite. Two groups can be recognized. The less radiogenic, namely Type $A$, (model ages of $300-400 \mathrm{Ma},{ }^{206} \mathrm{~Pb} /$ ${ }^{207} \mathrm{~Pb} \sim 1.15-1.16$ ) corresponds to high temperature deposits (Lac des Perches, Saulx), the vein of Heidenkopf, and the $\mathrm{Pb} / \mathrm{Sb}$ veins (Lepuix $\mathrm{Gy}$, Urbeis), while the more radiogenic includes all of the $\mathrm{F} / \mathrm{Ba}$ and $\mathrm{Ag} / \mathrm{Pb}$ veins, except Lusse. This group, namely Type $\mathrm{B}$, is characterised by model ages from $+30 \mathrm{Ma}$ to $-140 \mathrm{Ma}$ and ${ }^{206} \mathrm{~Pb} /{ }^{207} \mathrm{~Pb}$ ratios of about 1.19-1.20.

\begin{tabular}{|c|c|c|c|c|c|c|}
\hline Location & Mineral & $\begin{array}{l}{ }^{206} \mathrm{~Pb} / \\
{ }^{204} \mathrm{~Pb}\end{array}$ & $\begin{array}{l}{ }^{207} \mathrm{~Pb} / \\
{ }^{204} \mathrm{~Pb}\end{array}$ & $\begin{array}{l}{ }^{208} \mathrm{~Pb} / \\
{ }^{204} \mathrm{~Pb}\end{array}$ & $\begin{array}{l}{ }^{206} \mathrm{~Pb} / \\
{ }^{207} \mathrm{~Pb}\end{array}$ & $\begin{array}{l}{ }^{208} \mathrm{~Pb} / \\
{ }^{206} \mathrm{~Pb}\end{array}$ \\
\hline \multicolumn{7}{|c|}{ High temperature ore deposits } \\
\hline Lac des Perches & Ak & 17.964 & 15.546 & 37.918 & 1.156 & 2.111 \\
\hline Saulx & G & 18.120 & 15.547 & 37.798 & 1.165 & 2.086 \\
\hline $\begin{array}{l}\text { Sainte-Marie-aux- } \\
\text { Mines }\end{array}$ & G & 18.789 & 15.630 & 38.679 & 1.202 & 2.059 \\
\hline \multicolumn{7}{|l|}{ Mesothermal deposits } \\
\hline $\begin{array}{l}\text { La Croix } \\
\text { aux-Mines }\end{array}$ & G & 18.871 & 15.638 & 38.785 & 1.207 & 2.055 \\
\hline $\begin{array}{l}\text { La Croix } \\
\text { aux-Mines }\end{array}$ & G & 18.848 & 15.642 & 38.770 & 1.205 & 2.057 \\
\hline Remémont & G & 18.883 & 15.629 & 38.740 & 1.208 & 2.052 \\
\hline Lusse & G & 18.320 & 15.640 & 38.398 & 1.171 & 2.096 \\
\hline Urbéis & G & 18.182 & 15.603 & 38.110 & 1.165 & 2.096 \\
\hline Urbéis & $\mathrm{Bl}$ & 18.171 & 15.597 & 38.090 & 1.165 & 2.096 \\
\hline Moosch & G & 18.625 & 15.604 & 38.439 & 1.194 & 2.064 \\
\hline Lepuix-Gy & Z & 18.066 & 15.569 & 38.107 & 1.160 & 2.109 \\
\hline Lepuix-Gy & $\mathrm{P}$ & 18.080 & 15.570 & 38.137 & 1.161 & 2.109 \\
\hline Lepuix-Gy & $\mathrm{Bl}$ & 18.089 & 15.565 & 38.128 & 1.162 & 2.108 \\
\hline Charbes & Bert. & 18.370 & 15.735 & 38.837 & 1.167 & 2.114 \\
\hline \multicolumn{7}{|l|}{ Epithermal veins } \\
\hline Giromagny & G & 18.869 & 15.652 & 38.770 & 1.206 & 2.055 \\
\hline Giromagny & G & 18.844 & 15.642 & 38.750 & 1.205 & 2.056 \\
\hline Trémeures & G & 18.877 & 15.652 & 38.820 & 1.206 & 2.056 \\
\hline Maxonchamp & G & 18.788 & 15.632 & 38.695 & 1.202 & 2.060 \\
\hline Auxelles & G & 18.814 & 15.625 & 38.529 & 1.204 & 2.048 \\
\hline Mont-de-Vannes & G & 18.761 & 15.679 & 38.805 & 1.197 & 2.068 \\
\hline Mont-de-Vannes & G & 18.787 & 15.682 & 38.947 & 1.198 & 2.073 \\
\hline Heidenkopf & G & 18.169 & 15.576 & 38.148 & 1.166 & 2.100 \\
\hline
\end{tabular}


Fortunately, the discovery in 1549 of a silver-rich copper vein some kilometers south of Sainte-Marie-aux-Mines in the Neuenberg (the new mountain) by Schwartz Collin strongly stimulated production. Simultaneously, a new process, liquation, which consists in adding considerable amounts of lead to copper in order to increase silver extraction, was massively applied thus improving profitability. Nonetheless, after a thirty-year period of wealth, mining declined again due to wood shortages, augmentation of production costs, and the discovery of mines in the New World which provided silver for Europe. The Thirty Years' War during the first half of the 17th century led to plundering and occupation by Swedish, French and Imperial troops. Water rose in shafts and production collapsed again. At the beginning of the 18th century, mines reopened focusing on silver, lead, copper and a newly exploited metal: cobalt. However, infrastructures suffered from fires, floods and various accidents which damaged the fragile industrial balance. A new phase of recession occurred from the second half of the 18th century. This historical summary shows that local mining and smelting underwent several peaks and troughs during the 16th-18th century. Unfortunately, they are not all visible in our profile, except the revival in the 18th century which probably corresponds to lead fluxes peaking at about $11 \mathrm{mg} \mathrm{m}^{-2} \mathrm{y}^{-1}$ (Fig. 5a). A higher resolution sub-sampling and the establishment of a ${ }^{210} \mathrm{~Pb}$ chronology over the last century, extrapolated to depth, should improve the reconstruction of this past history. However, it must be kept in mind that, because they are formed from living plants, peat sequences are probably less efficient than laminated and undisturbed sediments at preserving high-frequency variations. In all cases, the slow industrial decline of the area, which was marked only by some attempts at exploitation during the 19th century, lingering on until World War II, explains the decrease in excess lead fluxes during the 19 th and 20th centuries. The low ${ }^{206} \mathrm{~Pb} /{ }^{207} \mathrm{~Pb}$ ratio ca 1.12 in the sub-surface horizon of Gazon-du-Faing (Fig. 5a) is clearly incompatible with local or regional mining (Fig. 6). In contrast, it illustrates the influence of unradiogenic lead added as an antiknock compound in gasoline from the end of the 20th century (Hamester et al., 1994; Monna et al., 1997; Chiaradia and Cupelin, 2000).

3.4.3.2. Rossely. The first signs of medieval contamination appear from the 12th-13th century in the Rossely core (Fig. 5b), in other words later than for Gazon-du-Faing. Although the medieval mining history is not well known in the district of Plancher-les-Mines, some sources seem to indicate that mining started in the area as early as the 12th century AD. The first written evidence dates from the 15th century, when the priests of Lure signed a lease for local metal exploitation. Later, during the 16th century, anthropogenic lead fluxes increased rapidly. At the beginning of the 16th century, the silver mines of Plancher-les-Mines were among the most productive in the Empire. The district furnished Guebwiller (Alsace, France) with silver for coinage. As in the case of the Sainte-Marie-aux-Mines district, the Thirty Years' War damaged local mining and smelting. Extraction and treatment works began again from the end of the 17th century, peaked during the 18th century and finally ceased in 1770. In the Rossely core, just as in the Gazon-du-Faing core, the peak of activity is clearly recorded (Fig. 5b), as is perhaps the slowing down which occurred during the first half of the 16th century. Except for the topmost samples which are less radiogenic, probably because of the influence of unradiogenic leaded gasoline, the lead isotopic compositions of the anthropogenic inputs remained steady at Rossely from the end of the 13th century to modern times, with ${ }^{206} \mathrm{~Pb} /{ }^{207} \mathrm{~Pb}$ ratios between 1.19 and 1.20 (Fig. 5b). These signatures suggest a mixture between the two types of galena recognized in the area: ${ }^{206} \mathrm{~Pb} /$ ${ }^{207} \mathrm{~Pb}=1.155-1.165$ and ${ }^{206} \mathrm{~Pb} /{ }^{207} \mathrm{~Pb}=1.194-1.204$ (see Fig. 1 for localisation and Fig. 6, details are given in Table 6), with however a paramount importance of the second end-member. Interestingly, the isotopic differences observed between the two cores from the 10th century onwards (Fig. 5a and b) confirm that atmospheric deposition at this period is mainly driven by local mining and smelting, rather than by inputs originating from remote sources. It is also noteworthy that excess lead fluxes appear to be much greater in magnitude at Rossely than at Gazon-du-Faing (Fig. 5a and b). It would nonetheless be false to conclude that exploitation at Plancher-les-Mines was more intense than at Sainte-Marie-aux-Mines. The peatland of Rossely is located leeward, at only $5-10 \mathrm{~km}$ from the main mines, while the Gazon-du-Faing peat was cored more than $20 \mathrm{~km}$ south of SainteMarie-aux-Mines (Fig. 1). Their situations with respect to supposed contamination sources are thus quite different in terms of distance from emissions and position with respect to dominant wind direction.

\subsection{Bismuth as possible proxy of metallurgy}

Bismuth is a metal that was often mistaken for lead in the past as they are chemically close. It may be associated as a by-product to some lead or copper ores, so that discrete but significant contamination has been observed as early as Greek/Phoenician times in Arctic ice (Krachler et al., 2009). In our case, from the medieval period and onwards, the Bi profile in the Gazon-du-Faing core exhibits some resemblance with $\mathrm{Pb}$, except that it begins to increase later and that it drops appreciably during the 14 th century (Fig. 4b). The resulting large variations in $\mathrm{Pb} / \mathrm{Bi}$ ratios between 100 and 850 appear to be smooth and closely related to those of ${ }^{206} \mathrm{~Pb} /{ }^{207} \mathrm{~Pb}$ ratios from the Middle Ages (see the simultaneous peaks at times T1 and T2 in Fig. 4b). This similitude strongly suggests that $\mathrm{Pb} / \mathrm{Bi}$ ratios might help in identifying the mineralizations exploited throughout history. As a matter of fact, the drastic rise in Bi contents from the 16th century AD onwards clearly matches mining works undertaken from 1549 in the Neuenberg, an area known to present substantial traces of Bi, while ores from Altenberg exploited earlier do not contain as much. During the Iron Age and Antiquity, high $\mathrm{Pb} / \mathrm{Bi}$ ratios are also detected (Fig. 4b). They present two top values corresponding to horizons dated at approximately $250 \mathrm{BC}$ and $50 \mathrm{AD}$. This feature, if it is confirmed, might be used in the future to trace the pollution deriving from long-range transport of contaminated particles across Europe.

\section{Conclusion}

The geochemical reading of lead signals archived in both cores is in very good agreement with the local archaeological and historical knowledge available for the two mining districts over the last millennium. This result clearly demonstrates the effectiveness of our approach. Even if the temporal resolution may appear rather poor to archaeologists and historians, it allows a rapid overview of local mining and smelting. Concerning the earliest periods, no mining or smelting was clearly identified before the 10th century. Geochemical anomalies recognized for the Iron Age and Antiquity were attributed to continental signals observed everywhere in Europe at those periods. A discrete anomaly was also noticed for the Bronze Age at the site of Rossely but its origin is not clear enough to identify local mining with confidence.

The present work also stresses the massive metal contamination in areas nowadays protected by the French government because of their remarkable biotopes. $\mathrm{Pb}$ concentrations reach respectively more than $100 \mu \mathrm{g} \mathrm{g}^{-1}$ and $500 \mu \mathrm{g} \mathrm{g}^{-1}$ in the sub-surface horizons of Gazon-duFaing and Rossely. These areas are located several kilometers away from former emission sources, so that the immediate surroundings of the former mines and smelters are in all probability much more heavily contaminated. Environmental studies should therefore be undertaken to assess the impact on present-day fauna and human health.

\section{Acknowledgements}

We wish to thank the Regional Council of Burgundy and the CNRS for their support, and funding for the Ph.D. thesis of Benoit Forel (CNRS BDI-R 2004.3 Bourgogne), as well as the ACR programme 
directed by Jean-François Piningre, which studies the Bronze Age in eastern France. We are grateful to anonymous reviewers who improved the manuscript by their judicious comments.

\section{References}

Alfonso, S., Grousset, F., Massé, L., Tastet, J.-P., 2001. A European lead isotope signal recorded from 6000 to 300 years BP in coastal marshes (SW France). Atmospheric Environment 35, 3595-3605.

Arnaud, F., Revel-Rolland, M., Bosch, D., Winiarski, T., Desmet, M., Tribovillard, N., Givelet, N., 2004. A 300 year history of lead contamination in northern French Alps reconstructed from distant lake sediment records. Journal of Environmental Monitoring 6, 448-456.

Baker, J., Peate, D., Waight, T., Meyzen, C., 2004. Pb isotopic analysis of standards and samples using a $\mathrm{Pb}-207-\mathrm{Pb}-204$ double spike and thallium to correct for mass bias with a double-focusing MC-ICP-MS. Chemical Geology 211, 275-303.

Bari, H., Benoit, P., Brugerolles, E., Fluck, P., Schoen, H., 1992. La mine mode d'emploi. La Rouge Myne de Sainct Nicolas de La Croix. Collection Découvertes Gallimard. 84 pp.

Baron, S., Lavoie, M., Ploquin, A., Carignan, J., Pulido, M., de Beaulieu, J.-L., 2005. Record of metal workshops in peat deposits: history and environmental impact on the Mont Lozere Massif, France. Environmental Science and Technology 39, 5131-5140.

Bindler, R., 2006. Mired in the past - looking to the future: geochemistry of peat and the analysis of past environmental changes. Global and Planetary Change 53, 209-221.

Bindler, R., Renberg, I., Rydberg, J., Andren, T., 2009. Widespread waterborne pollution in central Swedish lakes and the Baltic Sea from pre-industrial mining and metallurgy. Environmental Pollution 157, 2132-2141.

Brännvall, M.-L., Bindler, R., Renberg, I., Emteryd, O., Bartnicki, J., Billström, K., 1999. The medieval metal industry was the cradle of modern large-scale atmospheric lead pollution in Northern Europe. Environmental Science and Technology 33, 4391-4395.

Chiaradia, M., Cupelin, F., 2000. Behaviour of airborne lead and temporal variations of its source effects in Geneva (Switzerland): comparison of anthropogenic versus natural processes. Atmospheric Environment 34, 959-971.

De Vleeschouwer, F., Gerard, L., Goormaghtigh, C., Mattielli, N., Le Roux, G., Fagel, N., 2007. Atmospheric lead and heavy metal pollution records from a Belgian peat bog spanning the last two millennia: human impact on a regional to global scale. Science of the Total Environment 377, 282-295.

Dumontet, S., Lévesque, M., Mathur, S.P., 1990. Limited downward migration of pollutant metals $(\mathrm{Cu}, \mathrm{Z}, \mathrm{Ni}$, and $\mathrm{Pb})$ in acidic virgin peat soils near a smelter. Water Air Soil Pollution 49, 329-342.

Elbaz-Poulichet, F., Holliger, P., Martin, J.M., Petit, D., 1986. Stable lead isotopes ratios in major french rivers and estuaries. Science of the Total Environment 54, 61-76.

Faure, G., 1986. Principles of Isotope Geology. Wiley and sons, NewYork, USA.

Fluck, P., 2000. Sainte-Marie-aux-Mines. Les mines du rêve. Editions du patrimoine minier, p. 208.

Fluck, P., 2009. PCR Altenberg (Ste-Marie-aux-Mines, Haut-Rhin): fonderies et mines d'argent, Xe-XVIIe s., Les Actes du CRESAT, No. 6. Université de Haute-Alsace.

Fluck, P., Weil, R., 1975. Géologie des gîtes minéraux dans les Vosges: Mémoires du B.R. G.M., 87, p. 190.

Hamester, M., Stechmann, M., Steiger, M., Dannecker, W., 1994. The origin of lead in urban aerosols - a lead isotopic ratio study. Science of the Total Environment 146/ 147, 321-323.

Hong, S., Candelone, J.P., Patterson, C.C., Boutron, C.F., 1994. Greenland ice evidence of hemispheric lead pollution two millenia ago by Greek and Roman civilizations. Science 265, 1841-1843.

Jouffroy-Bapicot, I., Pulido, M., Galop, D., Monna, F., Ploquin, A., Baron, S., Petit, C., Lavoie, M. Beaulieu, J.-L., de Richard, H., 2007. Environmental impact of early palaeometallurgy: pollen and geochemical analysis. Vegetation History and Archaeobotany 16, 251-258.

Komárek, M., Ettler, V., Chrastný, C., Mihaljevič, M., 2008. Lead isotopes in environmental sciences: a review. Environment International 34, 562-577.

Krachler, M., Zheng, J., Fisher, D., Shotyk, W., 2009. Global atmospheric As and Bi contamination preserved in 3000 year old Arctic ice. Global Biogeochemical cycles 23, GB3011 7 pp..

Kylander, M.E., Weiss, D.J., Martínez Cortízas, A., Spiro, B., Garcia-Sanchez, R., Coles, B.J., 2005. Refining the pre-industrial atmospheric $\mathrm{Pb}$ isotope evolution curve in Europe using an 8000 year old peat core from NW Spain. Earth and Planetary Science Letters 240, 467-485.

Kylander, M.E., Weiss, D.J., Kober, B., 2009. Two high resolution terrestrial records of atmospheric $\mathrm{Pb}$ deposition from New Brunswick, Canada, and Loch Laxford, Scotland. Science of Total Environment 407, 1644-1657.

Le Roux, G., Aubert, D., Stille, P., Krachler, M., Kober, B., Cheburkin, A., Bonani, G., Shotyk, W., 2005. Recent atmospheric Pb deposition at a rural site in southern Germany assessed using a peat core and snowpack, and comparison with other archives. Atmospheric Environment 39, 6790-6801.

Lévêque, J., Phillippe, S., Baize, D., Monna, F., Haack, U., 2002. Utilisation des isotopes stables du plomb pour la détermination des sources de pollutions et l'étude de son transfert dans les sols contaminés. In: Baize, D., Tercé, M. (Eds.), « Les éléments traces métalliques dans les sols. Approches fonctionnelles et spatiales » INRA: coord., pp. 375-391.

MacKenzie, A.B., Pulford, I.D., 2002. Investigation of metal dispersal from a disused mine site at Tyndrum, Scotland, using concentration gradients and stable $\mathrm{Pb}$ isotope ratios. Applied Geochemistry 17, 1093-1103.

Marcoux, E., 1987. Isotopes du plomb et paragenèses métalliques. Traceurs de l'histoire des gîtes minéraux. Document du BRGM, No. 117. 289 pp.

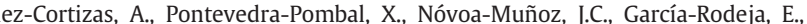
1997. Four thousand years of atmospheric $\mathrm{Pb}, \mathrm{Cd}$ and $\mathrm{Zn}$ deposition recorded by the ombrotrophic peat bog of Penido Vello (Northwestern Spain). Water Air Soil Pollution 100, 387-403.

Martínez-Cortizas, A., Garcia-Rodeja, E., Pontevedra Pombal, X., Nóvoa-Muñoz, J.C. Weiss, D., Cherbulin, A., 2002. Atmospheric Pb deposition in Spain during the last 4600 years recorded by two ombrotrophic peat bogs and implications for the use of peat as archive. Science Total Environment 292, 33-44.

Mighall, T.M., Abrahams, P.W., Grattan, J.P., Hayes, D., Timberlake, S., Forsyth, S., 2002. Geochemical evidence for atmospheric pollution derived from prehistoric copper mining at Copa Hill, Cwmystwyth, mid-Wales, UK. Science of the Total Environment 292, 69-80.

Mighall, T.M., Timberlake, S., Foster, I.D.L., Krupp, E., Singh, S., 2009. Ancient copper and lead pollution records from a raised bog complex in Central Wales, UK. Journal of Archaeological Science 36, 1504-1515.

Mihaljevič, M., Zuna, M., Ettler, V., Šebek, O., Strnad, L., Goliáš, V., 2006. Lead fluxes, isotopic and concentration profiles in a peat deposit near a lead smelter (Pribram, Czech Republic). Science of the Total Environment 372, 334-344.

Monna, F., Lancelot, J., Croudace, I., Cundy, A.B., Lewis, T., 1997. Pb isotopic signature of urban air in France and in UK: implications on Pb pollution sources. Environmental Science and Technology 31, 2277-2286.

Monna, F., Loizeau, J.-L., Thomas, B.A., Guéguen, C., Favarger, P.-Y., 1998. Pb and Sr isotope measurements by inductively coupled plasma-mass spectrometer: efficient time management for precise improvement. Spectrochimica Acta B 59, 1317-1333.

Monna, F., Loizeau, J.-L., Thomas, B., Guéguen, C., Favarger, P.-Y., Losno, R., Dominik, J., 2000. Noise identification and sampling frequency determination for precise isotopic measurements by quadrupole-based inductively coupled plasma mass spectrometry. Analusis 28, 750-757.

Monna, F., Petit, C., Guillaumet, J.-P., Jouffroy-Bapicot, I., Blanchot, C., Dominik, J., Losno, R., Richard, H., Lévêque, J., Chateau, C., 2004a. History and environmental impact of mining activity in Celtic Aeduan territory recorded in a peat-bog (Morvan, France). Environmental Science and Technology 38, 657-673.

Monna, F., Galop, D., Carozza, L., Tual, M., Beyrie, A., Marembert, F., Chateau, C., Dominik, J., Grousset, F.E., 2004b. Environmental impact of early Basque mining and smelting recorded in a high ash minerogenic peat deposit. Science of the Total Environment 327, 197-214.

Monna, F., Poujol, M., Annegarn, H., Losno, R., Coetze, H., Dominik, J., 2006. Origin of atmospheric lead in Johannesburg, South Africa. Atmospheric Environment 40, 6554-6566.

Nriagu, J.O., 1983. Lead and Lead Poisoning in Antiquity. Wiley, New York.

Nriagu, J.O., 1996. A history of global metal pollution. Science 272, 223-224.

Renberg, I., Bindler, R., Brännvall, M.-L., 2001. Using the historical atmospheric leaddeposition record as a chronological marker in sediment deposits in Europe. Holocene 11, 511-516.

Rosman, K.J.R., Chisholm, W., Hong, S.M., Candelone, J.P., Boutron, C.F., 1997. Lead from Carthaginian and Roman Spanish mines isotopically identified in Greenland ice dated from $600 \mathrm{BC}$ to $300 \mathrm{AD}$. Environmental Science and Technology 31, 3413-3416.

Settle, D., Patterson, C., 1980. Lead in albacore: guide to lead pollutions in Americans. Science 207, 1167-1176.

Shotyk, W., 1996a. Peat bog archives of atmospheric metal deposition: geochemical evaluation of peat profiles, natural variations in metal concentrations, and metal enrichment factors. Environmental Review 4, 149-183.

Shotyk, W., 1996b. Natural and anthropogenic enrichments of As, Cu, Pb, Sb, and Zn in ombrotrophic vs. minerotrophic peat bog profiles, Jura Mountains, Switzerland. Water Air Soil Pollution 90, 375-405.

Shotyk, W., 2002. The chronology of anthropogenic, atmospheric $\mathrm{Pb}$ deposition recorded by peat cores in three minerogenic peat deposits from Switzerland. Science of the Total Environment 292, 19-31.

Shotyk, W., Cheburkin, A.K., Appleby, P.G., Fankhauser, A., Kramers, J.D., 1997. Lead in three peat bog profiles, Jura Mountains, Switzerland: enrichment factors, isotopic composition, and chronology of atmospheric deposition. Water Air and Soil Pollution 100, 297-310.

Shotyk, W., Weiss, D., Appleby, P., Cherbukin, A., Frei, R., Gloor, M., Kramer, J., Reese, S. Van der Knaap, W., 1998. History of atmospheric lead deposition since 12, $37014 \mathrm{C}$ yr BP from a peat bog, Jura Mountains, Switzerland. Science 281, 1635-1640.

Shotyk, W., Goodsite, M.E., Roos-Barraclough, F., Frei, R., Heinemeier, J., Asmund, G., Lohse, C., Hansen, T.S., 2003. Anthropogenic contributions to atmospheric $\mathrm{Hg}, \mathrm{Pb}$ and As accumulation recorded by peat cores from southern Greenland and Denmark dated using the 14C "bomb pulse curve". Geochimica Cosmochimica Acta 67, 3991-4011.

Vile, M.A., Wieder, R.K., Novak, M., 1999. Mobility of Pb in Sphagnum-derived peat. Biogeochemistry 45, 35-52.

Wedepohl, K.H., 1995. The composition of the continental crust. Geochimica et Cosmochimica Acta 59, 1217-1232.

Weiss, D., Shotyk, W., Appleby, P.G., Cheburkin, A.K., Kramers, J.D., 1999. Atmospheric $\mathrm{Pb}$ deposition since the Industrial Revolution recorded by five Swiss peat profiles: enrichment factors, fluxes, isotopic composition, and sources. Environmental Science and Technology 33, 1340-1352.

Weiss, D., Shotyk, W., Boyle, E.A., Kramers, J.D., Appleby, P.G., Cheburkin, A.K., 2002. Comparative study of the temporal evolution of atmospheric lead deposition in Scotland and eastern Canada using blanket peat bogs. Science Total Environment 292, 7-18. 